\title{
Conformal symmetry and light flavor baryon spectra
}

\author{
M. Kirchbach, C. B. Compean \\ ${ }^{2}$ Instituto de Física, \\ Universidad Autónoma de San Luis Potosí, \\ Av. Manuel Nava 6, San Luis Potosí, \\ S.L.P. 78290, México
}

(Dated: June 10, 2022) 


\begin{abstract}
The degeneracy among parity pairs systematically observed in the $N$ and $\Delta$ spectra is interpreted to hint on a possible conformal symmetry realization in the light flavor baryon sector in line with $\mathrm{AdS}_{5} / \mathrm{CFT}_{4}$. The case is made by showing that all the observed $N$ and $\Delta$ resonances with masses below $2500 \mathrm{MeV}$ distribute fairly well each over the first levels of a unitary representation of the conformal group, a representation that covers the spectrum of a quark-diquark system, placed directly on a conformally compactified Minkowski spacetime, $\mathbf{R}^{1} \otimes S^{3}$, as approached from the the $\mathrm{AdS}_{5}$ cone. The free geodesic motion on the $S^{3}$ manifold is described by means of the scalar conformal equation there, which is of the Klein-Gordon type. The equation is then gauged by the "curved" Coulomb potential that has the form of a cotangent function. Conformal symmetry is not exact, this because the gauge potential slightly modifies the conformal centrifugal barrier of the free geodesic motion. Thanks to this, the degeneracy between $P_{11}-S_{11}$ pairs from same level is relaxed, while the remaining states belonging to same level remain practically degenerate. The model describes the correct mass ordering in the $P_{11}-S_{11}$ pairs through the nucleon spectrum as a combined effect of the above conformal symmetry breaking, on the one side, and a parity change of the diquark from a scalar at low masses, to a pseudoscalar at higher masses, on the other. The quality of the wave functions is illustrated by calculations of realistic mean-square charge radii and electric charge form-factors on the examples of the proton, and the protonic $P_{11}(1440)$, and $S_{11}(1535)$ resonances. The scheme also allows for a prediction of the dressing function of an effective instantaneous gluon propagator from the Fourier transform of the gauge potential. We find a dressing function that is finite in the infrared and tends to zero at infinity.
\end{abstract}

PACS numbers: 12.39.Jh, 24.85.+p

Keywords: AdS/CFT, higher spins, electric mean square charge radii 


\section{INTRODUCTION}

Understanding the systematics of high-spin states is among the serious challenges in quark spectroscopy [1],[2]. This because the number of resonances predicted by the traditional quark models based upon the full Hilbert space of six spin-flavor degrees of freedom and the lowest symmetry of the radial wave functions, the rotational invariance, [3] significantly exceeds the number of the states observed so far [4]. The resonance deficit, termed to as "missing" states, is still awaiting for explanation. Quark-diquark (q-(qq)) models [5] based on a diquark with limited angular momentum values, carry reduced spin-flavor degrees of freedom, and are obvious candidates for providing a lesser number of "missing" states, an option taken into consideration by several authors [6], [7]. Additional restrictions on the quantum numbers of the $q$ - $(q q)$ excitations can come from imposing on the spatial wave functions a symmetry higher than $\mathrm{SO}(3)_{L}$. A natural candidate would be the, admittedly approximate, global conformal symmetry of the QCD Lagrangian in the light flavor sector. It is the goal of the present work to examine consequences of conformal symmetry for the systematics of the $N$ and $\Delta$ spectra in constructing radial wave functions of a $q-(q q)$ system in accord with that very symmetry. The aim is to pick up from the full Hilbert space isospin by isospin those $N$ and $\Delta$ resonances whose quantum numbers would fit into an irreducible representation of the conformal group (termed to as conformal band). The observation of these states can then serve as a signature for conformal symmetry realization in the infrared. Below we design such a model in reference to the $\mathrm{AdS}_{5} / \mathrm{CFT}_{4}$ concept. We shall see that the model allows to interpret the systematically observed degeneracy among parity pairs in the $N$ and $\Delta$ spectra as a hint on a possible realization of conformal symmetry in light flavor baryon spectra. Implementations of conformal symmetry and the AdS/CFT concept to hadron physics have been pioneered in refs. [8], [9] within the framework of lightfront QCD. Further interesting studies can be found, among others, in refs. [10], [11], [12], [13]. Specifically in refs. [8],[9], a conformally invariant Hamiltonian has been successfully employed in the construction of spatial wave functions for both mesons and baryons. We here implement conformal symmetry into a quark Hamiltonian in a position space of a finite volume. This is achieved in placing the $q-(q q)$ system directly on the $\mathrm{AdS}_{5}$ boundary, which is the $\mathrm{AdS}_{5}$ cone, considered as conformally compactified to $S^{1} \otimes S^{3}[14]$, or, $\mathbf{R}^{1} \otimes S^{3}$, at a microscopic scale [15]. According to [15], correlation functions of $\mathrm{CFT}_{4}$ on regular Minkowski 
spacetime, $\mathcal{M}=\mathbf{R}^{1+3}$, can be analytically continued to the full Einstein universe, this because $\mathbf{R}^{1+3}$ can be conformally mapped on $\mathbf{R}^{1} \otimes S^{3}$. The implication of this important observation is that each state of the CFT on $\mathbf{R}^{1} \otimes S^{3}$ can be brought into unique correspondence with a state of the brane theory on $A d S_{5} \otimes S^{5}$. Consequences on thermal states have been worked out in ref. [16]. We here examine consequences for the systematics of the $N$ and $\Delta$ spectra. The paper is organized as follows. In the next section we highlight the procedure of conformal compactification of Minkowski spacetime along the line of ref. [17] and present the conformal equation which will be applied to a quark-diquark (q-qq) model of light baryons in section 3. There, we calculate the $N$ and $\Delta$ spectra, the mean square charge radii and the corresponding electric charge form-factors of some of the excited states. Section 4 is devoted to the design of a dressing function of an (instantaneous) effective gluon propagator as a Fourier transform of the gauge potential on $S^{3}$. The paper closes with brief conclusions.

\section{CONFORMAL COMPACTIFICATION OF THE $\operatorname{ADS}_{5}$ CONE TO $S^{1} \otimes S^{3} \sim \mathbf{R}^{1} \otimes$ $S^{3}$, WAVE EQUATIONS, AND COTANGENT CONFINEMENT POTENTIAL}

Applications of brane theory to hadron physics have acquired considerable attention in recent times. Such a possibility arose in effect of the intriguing observation [14]-[15] that the asymptotic horizon geometry of the Dirichlet three-brane (D3) of the IIB superstring in ten dimensions considered on a $A d S_{5} \otimes S^{5}$ background, admits a superalgebra that is identical to the super-conformal algebra of the corresponding four dimensional world-volume field theory when gravity is decoupled. The D3 brane theory has $S U(2,2 / 4)$ as underlying superconformal symmetry whose bosonic isometry $S U(2,2) \otimes S U(4)$, is locally isomorphic to $S O(2,4) \otimes S O(6)[18]$. This group happens to coincide with the isometry group of the corresponding asymptotic horizon background, $A d S_{5} \otimes S^{5}$, a mathematical coincidence that was suggestive of a duality between supergravity around the horizon background, on the one side, and superconformal brane dynamics, on the other. In such a type of strong-weak duality, one expects the fundamental supergraviton degrees of freedom to show up as bound states in the non-perturbative regime of the corresponding world-volume theory. As a reminder, D3-branes solve the ten-dimensional supergravity equations of motion and in having a $(1+3)$ dimensional world volume, are surrounded by six transverse dimensions. In polar coordinates five of the $S O(6)$ dimensions are accounted for in terms of the angular coordinates 
parameterizing the $S^{5}$ hypersphere, while the sixth hyper radial coordinate is to become holographic with respect to $A d S_{5}$ in the near horizon geometry. There, the branes lowenergy effective description, the Yang-Mills theory, becomes equivalent to type II B string, a reason for which a duality between $A d S_{5}$ and $4 \mathrm{~d}$ supersymmetric Yang-Mills theory has been conjectured by Maldacena [19]. Maldacena's conjecture [19] woke up expectations that zero temperature super Yang-Mills theory residing in the conformal $A d S_{5}$ boundary is likely to capture some of the essential features of high-temperature three-dimensional QCD [20]-[24]. Within this context, testing AdS/CFT reduces to the calculation of observables within that very framework and their comparison to the corresponding Lattice results in $3 \mathrm{~d}$ QCD. The duality between D3-brane bulk supergravity and super Yang-Mills open string theories on the conformal boundary of $A d S_{5}$ space-time implies that each CFT state, (among them the QCD states in the light flavor sector) can be put in correspondence to a state in the supergravity approximation to string theory on $A d S_{5} \otimes S^{5}$ meaning that spectra in both theories should come out same. As long as the isometry group of the conformal $A d S_{5}$ boundary is the conformal group $S O(2,4)$, the requirement is that the quantum states in the theory on that boundary should populate $S O(2,4)$ unitary representations [20]. This is an essential restriction which strongly limits the number of theories in line with the AdS/CFT and is suggestive of the construction of quark models that respect global conformal invariance. Conformal symmetry is independently to a good approximation a global symmetry of the QCD Lagrangian in the light-flavor sector, a reason for which one can expect spectroscopic data on the light flavor baryons, the nucleon and the $\Delta$, to be especially appropriate in examining the AdS/CFT concept. The implementation of conformal symmetry by a quark model has to combine with the confinement phenomenon, whose description is one of the major goals in the physics of hadrons. Confinement implies exclusion of scattering states and favoring discrete bound states alone. Putting systems on finite volumes is a standard strategy of spectrum discretization [25]. There is a variety of geometries appropriate for preserving the conformal symmetry by the resulting Hamiltonians, the three dimensional sphere, $S^{3}$, being one of them [15], [25], [17]. It has been shown that a geometry containing $S^{3}$, such as the $S^{1} \otimes S^{3}$ manifold, can be approached departing directly from $\mathrm{AdS}_{5}$ [14], [21], [17]. The five dimensional manifold $\mathrm{AdS}_{5}$ is defined as a $\mathbf{R}^{2+4}$ subspace according to

$$
u^{2}+v^{2}-x_{1}^{2}-x_{2}^{2}-x_{3}^{2}-x_{4}^{2}=\rho^{2},
$$


where $\rho$ is a fixed parameter. The boundary at infinity of this space is identified with the $A d S_{5}$ cone

$$
u^{2}+v^{2}-x_{1}^{2}-x_{2}^{2}-x_{3}^{2}-x_{4}^{2}=0 .
$$

Flat four-dimensional (4d) Minkowski space time can then be thought of as the intersection of the null hyperplane $v^{2}-x_{4}^{2}=0$ with the $A d S_{5}$ cone,

$$
v^{2}-x_{4}^{2}=u^{2}-x_{1}^{2}-x_{2}^{2}-x_{3}^{2}=0
$$

in which case $u$ and $\vec{x}=\operatorname{column}\left(x_{1}, x_{2}, x_{3}\right)$ in turn assume the róles of time and position vector in $\mathbf{R}^{1+3}$, respectively. A quark system placed on such a $5 \mathrm{~d}$ cone can then be described by means of the light-front formalism. This path has been taken by refs. [8], [9] and culminated to the holographic light-front QCD.

The path taken by the present investigation is rather to consider Minkowski space as emerging from an $\mathrm{AdS}_{5}$ cone alternatively patterned after,

$$
u^{2}+v^{2}=x_{1}^{2}+x_{2}^{2}+x_{3}^{2}+x_{4}^{2}=R^{2}, \quad R \neq 0
$$

in which case it compactifies to $\mathcal{M}^{*(1+3)}=S^{1} \otimes S^{3}$. The $S^{1} \otimes S^{3}$ manifold then describes the particular set of $\mathbf{R}^{2+4}$ null rays associated with eq. (4). As long as the isometry group, $S O(2) \otimes S O(4)$, of $S^{1} \otimes S^{3}$, is a subgroup of the conformal group $S O(2,4)$ of regular $(1+3)$ Minkowski space, $\mathcal{M}$, i.e. $S O(2) \otimes S O(4) \subset S O(2,4)$, eq. (4) is also referred to as conformal compactification of Minkowski space time. Now one can parametrize the $S^{1} \otimes S^{3}$ manifold by the four angles $\tau, \chi, \theta, \varphi$ in accordance with

$$
\begin{array}{ll}
u+i v=R e^{i \tau} & x_{1}+i x_{2}=R \sin \chi \sin \theta e^{i \varphi}, \quad x_{3}=R \sin \chi \cos \theta, \\
x_{4}^{2}+\mathbf{r}^{2}=R^{2}, \quad r=|\mathbf{r}|=R \sin \chi, \quad \chi=\sin ^{-1} r \sqrt{\kappa}, \quad \kappa=\frac{1}{R^{2}},
\end{array}
$$

where $R$ is the $S^{3}$ hyper-radius, and $\kappa$ the curvature. According to ref. [17], this map takes at a microscopic scale the flat space Minkowski metric to the metric of Einstein's $\mathbf{R}^{1} \otimes S^{3}$ cylinder,

$$
d s^{2}=\Omega^{-2}\left(-\mathrm{d} \tau^{2}+\mathrm{d} \chi^{2}+\sin ^{2} \chi\left(\mathrm{d} \theta^{2}+\sin ^{2} \theta \mathrm{d} \varphi^{2}\right)\right)
$$

with $\Omega$ being the conformal factor. In this way, one establishes the relationship, $S^{1} \otimes$ $S^{3} \simeq \mathbf{R}^{1} \otimes S^{3}$. 


\section{A. Free geodesic motion on $S^{3}$ and the conformal free rigid rotor.}

Within the metric of eq. (6), and the conformal factor being absorbed by the wave functions, the following conformally invariant massless scalar field equation has been found in ref. [17], [26]

$$
-\hbar^{2} \widehat{\square} \psi+\mu^{2} \psi=0
$$

Here, $\mu^{2}$ is the conformal constant (Ricci scalar) [17], $\widehat{\square}$ stands for the angular part of the 4d Laplace-Beltrami operator, which we here choose to express in terms of $\mathcal{K}^{2}$, and $\mathbf{L}^{2}$, the operators of the squared four- and three dimensional angular momenta as,

$$
\begin{aligned}
\hat{\square} & =-\frac{1}{R^{2}} \frac{\partial^{2}}{\partial \tau^{2}}-\frac{1}{R^{2}} \mathcal{K}^{2}, \\
-\mathcal{K}^{2} & =\left[\frac{1}{\sin ^{2} \chi} \frac{\partial}{\partial \chi} \sin ^{2} \chi \frac{\partial}{\partial \chi}-\frac{\mathbf{L}^{2}(\theta, \varphi)}{\sin ^{2} \chi}\right] .
\end{aligned}
$$

Furthermore, $\chi \in[0, \pi]$ is the second polar angle on $S^{3}$. The $\mathcal{K}^{2}$ eigenstates, $\mid K l m>$, are well known to belong to irreducible $S O(4)$ representations of the type $\left(\frac{K}{2}, \frac{K}{2}\right)$, and the quantum numbers, $K, l$, and $m$ define the eigenvalues of the respective four-, three- and two-dimensional angular momentum operators upon the states [27]. The $\mathcal{K}^{2}$ eigenvalues, $\lambda_{K}$, upon $\mid K l m>$, are known too and given by,

$$
\begin{array}{cl}
\mathcal{K}^{2}|K l m\rangle=\lambda_{K}|K l m\rangle, & \lambda_{K}=K(K+2), \quad|K l m\rangle \in\left(\frac{K}{2}, \frac{K}{2}\right), \quad K \in[0, \infty), \\
\mathbf{L}^{2}|K l m\rangle=l(l+1)|K l m\rangle, & \left.L_{z}|K l m>=m| K l m\right\rangle, \\
l \in[0, K], & m \in[-l, l] .
\end{array}
$$

The infinite series of solutions of eq. (9) constitute an $\infty$ d unitary representation of the conformal group that has been built up from the eigenstates of its little group, $S O(4)_{K}$ [18], [28]. This irrep will be frequently termed to as a "conformal band". Therefore, the conformal symmetry aspect of AdS/CFT is adequately captured by the $\mathcal{K}^{2}$ eigenvalue problem, which in this fashion qualifies as a suitable departure point toward the description of conformal excitation modes in two-body systems.

Independently, conformal symmetry is also to a good approximation global symmetry of the QCD Lagrangian in the light flavor sector, one more reason why employing the conformally invariant AdS/CFT scenario from above in modeling $N$ and $\Delta$ excitations should 
be of interest. Upon factorizing the $\tau$ dependence of the solution to eq. (77) as $\exp \left(i \frac{\mathcal{E}}{\hbar \sqrt{\kappa}} \tau\right)$, one arrives at the following angular equation on $S^{3}$,

$$
\left[\kappa \hbar^{2} \mathcal{K}^{2}-\mathcal{E}^{2}+\mu^{2}\right] \mathcal{S}(\chi)=0
$$

Though in angular variables, algebraically the conformal eq. (7), has the form of a KleinGordon equation which provides a relativistic description of the free geodesic motion on $S^{3}$ in terms of the eigenvalue problem of the squared four dimensional angular momentum. The spectrum of eq. (10) reads,

$$
\mathcal{E}_{K}^{2}-\mu^{2}=\kappa \hbar^{2} K(K+2)
$$

and represents no more but what one could term to as the spectrum of the "conformal free rigid rotor". On the unit sphere, $\kappa=1$, the solutions contain the Gegenbauer polynomials. The normalized total angular functions for this case, i.e. the $\mathcal{K}^{2}$ eigenstates in eqs. (9), (10) are then given by,

$$
\mid K l m>=\mathcal{S}(\chi) Y_{l}^{m}(\theta, \varphi) \longrightarrow N_{K l m} \sin ^{l} \chi C_{K-l}^{l+1}(\cos \chi) Y_{l}^{m}(\theta, \varphi) \equiv Y_{K l m}(\chi, \theta, \varphi)
$$

where $Y_{K l m}(\chi, \theta, \varphi)$ are well known 4d hyper-spherical harmonics.

As a more specialized spectroscopic reading to eqs. (10), one can say that the $\mathcal{K}^{2}$ eigenvalue problem has $S O(4)$ as potential algebra, and the conformal group as dynamical symmetry.

Had one started instead with $\mathrm{AdS}_{4}$, one would have ended up with the spectrum of the relativistic $3 \mathrm{~d}$ free rigid rotor, $E^{2}-\mu^{2}=\hbar^{2} \kappa l(l+1)$. Such a spectrum may be associated with Regge trajectories [29], a reason for the frequently preferred plots of resonance excitations on a mass ${ }^{2} / l$ grid beyond the strict S-matrix concept of Regge trajectories [10], [30].

In the next section we shall introduce an interaction in eq. (10).

\section{B. The conformal interacting rigid rotor.}

The next step is introducing interaction on $S^{3}$ in such a manner as to respect the conformal symmetry of the spectrum of the free geodesic motion. From potential theory it is known that such an interaction has to satisfy the Laplace-Beltrami equation on the manifold 
under consideration, meaning that it has to be a harmonic function there [31]. Harmonic functions are known for their property to respect the symmetry of the Laplacian which is the conformal symmetry of the respective $d$-dimensional space. Specifically on $S^{3}$, it has been known for a long time [32], [33] that cot $\chi$, occasionally termed to as "curved" Coulomb potential [36], is a harmonic function and suitable as a conformal potential. According to the $S^{3}$ parametrization in eq. (5) the potential under consideration is of finite range,

$$
\cot \chi=\frac{x_{4}}{r}, \quad r \in[0, R], \quad x_{4} \in[-R, R]
$$

and describes interactions on $S^{3}$ in their projection onto the equatorial plane, a $3 \mathrm{~d}$ position space of a finite volume. In due course we shall reveal importance of the finite range of the confinement potential on various spectroscopic observables in the baryon sector. The conformal character of the cotangent on $S^{3}$ is independently illustrated by the fact that it also describes an exact string solution corresponding to a D3 brane with transverse dimensions conformally wrapped over $S^{3}$, a result due to refs. [37], [38]. There, a broad class of exact string solutions have been constructed by wrapping transversal dimensions of fundamental strings over curved spaces and solving the corresponding curved space Laplace-Beltrami equations for harmonic functions. Specifically on $S^{3}$, the harmonic function $K(\chi)$ (in the notations of ref. [37]) obtained as a solution of the 4d Laplace-Beltrami equation,

$$
\widehat{\square} K(\chi)=0, \quad K(\chi)=\bar{a}+m \cot \chi
$$

has been shown to define the field of a conformal string solution according to,

$$
d s^{2}=\mathrm{d} u \mathrm{~d} v+K(\chi) \mathrm{d} u^{2}+\mathrm{d} \chi^{2}+\sin ^{2} \chi\left(\mathrm{d} \theta^{2}+\sin ^{2} \theta \mathrm{d} \varphi^{2}\right)
$$

For all these reasons, the cotangent function presents itself as suited for playing the part of a conformal potential on the $S^{3}$ space of finite volume. This curved space potential should not be confused with the flat space Wilson loop potential generated by $K(\chi)$ in the surrounding infinite $\mathbf{R}^{1+3}$ space.

A further and independent motivation in favor of employing the cotangent potential in quark models is provided by the observation [35] that the lowest terms in its Taylor expansion coincide with a Coulombic+linear (Cornell) potential. This is easiest to illustrate by the simplistic $\chi=\frac{r}{R} \pi$ parametrization (commonly used in super-symmetric quantum mechanics) 
for which

$$
-\cot \frac{r}{R} \pi=-\frac{d}{r}+\frac{1}{3} \frac{r}{d}+\frac{r^{3}}{45 d^{3}}+\frac{2 r^{5}}{945 d^{3}}+\ldots, \quad \text { with } \quad d=\frac{R}{\pi},
$$

holds valid. The Cornell potential [39] has been predicted by lattice QCD simulations [40], on the one side, and has been also independently confirmed within the AdS/CFT context where it emerges as a soft-wall Wilson loop potential [19], [41]. Its inverse distance part is associated with the one gluon exchange of the perturbative regime, while the linear term is viewed to describe the flux-tube interactions of the non-perturbative regime. In order to approach the regime of the asymptotic freedom, one needs to extend the Cornell potential by corrections that account for more complicated non-perturbative processes. Such have been systematically explored within the topological approach in refs. [42]. Within this context, the terms in eq. (16) beyond the first two could be viewed as a particular phenomenological parametrization of non-perturbative corrections beyond the flux-tube mechanism. As an advantage of such a parametrization we wish to mention the exact solubility of the resulting potential.

Therefore, the cotangent potential on $S^{3}$, besides being congruent with conformal symmetry in $\mathrm{AdS}_{5} / \mathrm{CFT}_{4}$, also adequately captures the dynamical aspects of QCD, a twofold advantage that makes it attractive to applications in hadron spectroscopy.

We shall introduce this very potential as a gauge interaction in eq. (8) by means of the replacement,

$$
i \hbar \sqrt{\kappa} \frac{\partial}{\partial \tau} \rightarrow i \hbar \sqrt{\kappa} \frac{\partial}{\partial \tau}+2 G \sqrt{\kappa} \cot \chi-\bar{a}
$$

where we parametrized $m$ in eq. (14) as $m=-2 G \sqrt{\kappa}$. Upon factorizing the $\tau$ dependence of the total wave function as $\exp \left(-i \frac{E}{\hbar \sqrt{\kappa}} \tau\right)$, and after some algebraic manipulations, the interacting Klein-Gordon equation can be cast into the following form,

$$
\begin{aligned}
\left(-\kappa \hbar^{2} \frac{\mathrm{d}^{2}}{\mathrm{~d} \chi^{2}}+U_{l}(\kappa, \chi)\right. & \left.-2 G \sqrt{\kappa}(2 E-2 \bar{a}) \cot \chi-(2 G \sqrt{\kappa})^{2} \csc ^{2} \chi\right) \Psi(\chi) \\
= & {\left[(E-\bar{a})^{2}-\mu^{2}-\left(\bar{a}^{2}+(2 G \sqrt{\kappa})^{2}\right)\right] \Psi(\chi) . }
\end{aligned}
$$


The second term on the l.h.s of this equation,

$$
U_{l}(\chi, \kappa)=h^{2} \kappa l(l+1) \csc ^{2} \chi
$$

is the centrifugal barrier of the free geodesic motion on $S^{3}$. Equation (18) has been obtained in making use of the peculiarity of the cotangent function to reproduce itself, and the $S^{3}$ centrifugal barrier, upon squaring,

$$
(\bar{a}+m \cot \chi)^{2}=\bar{a}^{2}-m^{2}+2 \bar{a} m \cot \chi+m^{2} \csc ^{2} \chi .
$$

Therefore, the $\csc ^{2}$ terms arising upon the substitution of eqs. (17) in eq. (8) can be absorbed by the $S^{3}$ centrifugal barrier. Indeed, introducing the new constant,

$$
\begin{aligned}
\alpha(l) & =-\frac{1}{2}+\sqrt{\left(l+\frac{1}{2}\right)^{2}-\frac{(2 G)^{2}}{\hbar^{2}}}=l+\Delta l, \\
\Delta l & \approx-\frac{1}{2} \frac{(2 G)^{2}}{\hbar^{2}} \frac{1}{l+\frac{1}{2}},
\end{aligned}
$$

allows one to rewrite eq. (18) equivalently to,

$$
\begin{gathered}
{\left[-\kappa \hbar^{2} \frac{\mathrm{d}^{2}}{\mathrm{~d} \chi^{2}}+\mathcal{V}(\chi)\right] \Psi(\chi)=\left((E-\bar{a})^{2}-c_{0}\right) \Psi(\chi),} \\
\mathcal{V}(\chi)=-2 \beta \cot \chi+\bar{U}_{l}(\chi, \kappa), \quad \bar{U}_{l}(\chi, \kappa)=\hbar^{2} \kappa \alpha(l)(\alpha(l)+1) \csc ^{2} \chi \\
\beta=2 G \sqrt{\kappa}(E-\bar{a}), \quad c_{0}=\mu^{2}-\hbar^{2} \kappa+\bar{a}^{2}+(2 G \sqrt{\kappa})^{2} .
\end{gathered}
$$

The positive sign in front of the square root in eq. (21) ensures that $\bar{U}_{l}(\chi, \kappa)$ approaches the $S^{3}$ centrifugal barrier of the free geodesic motion in the $G \rightarrow 0$ limit,

$$
\bar{U}_{l}(\chi, \kappa) \stackrel{G \rightarrow 0}{\longrightarrow} U_{l}(\chi, \kappa) .
$$

Upon a suitable variable change, differential equations of the type in (22) have been shown in refs. [34], [43] to reduce to one of the forms of the hyper-geometric equation whose solutions can be expressed in closed form in terms of the non-classical Romanovski polynomials (here denoted by $\left.R_{n}^{(a, b)}(\cot \chi)\right)$.

\section{Wave functions, spectrum and kinetic fine structure splittings}

The resulting explicit formula for the wave functions then emerges as,

$$
\begin{array}{r}
\Psi_{K l}(\chi)=N_{K l} e^{-a \chi}(\sin \chi)^{K+1+\Delta l} R_{n}^{(a, b)}(\cot \chi), \\
a=\frac{2 G(E-\bar{a})}{\sqrt{\kappa} \hbar^{2}(K+1+\Delta l)}, \quad b=-(K+1+\Delta l), \quad K=n+l,
\end{array}
$$


where $N_{K l}$ are normalization constants. Correspondingly, the algebraic equation for the energy takes the form,

$$
(E-\bar{a})^{2}=\frac{c_{0}+\hbar^{2} \kappa(K+1+\Delta l)^{2}}{1+\frac{4 G^{2}}{\hbar^{2}(K+1+\Delta l)^{2}}} .
$$

The expansion of the latter equation to leading order in $\Delta l$ reads,

$$
(E-\bar{a})^{2} \approx\left(E_{(K+1)}-\bar{a}\right)^{2}+\Delta E_{(K+1)}^{(1)}(\Delta l)
$$

where the $l$ independent piece,

$$
\left(E_{(K+1)}-\bar{a}\right)^{2}=\frac{c_{0}+\hbar^{2} \kappa(K+1)^{2}}{1+\frac{4 G^{2}}{\hbar^{2}(K+1)^{2}}},
$$

can be viewed as an unperturbed degeneracy energy. The difference, $(E-\bar{a})^{2}-\left(E_{(K+1)}-\right.$ $\bar{a})^{2}=\Delta E_{(K+1)}^{(1)}(\Delta l)$, then calculates as

$$
\Delta E_{(K+1)}^{(1)}(\Delta l)=2 \Delta l\left(\frac{\hbar^{2} \kappa(K+1)}{1+\frac{4 G^{2}}{\hbar^{2}(K+1)^{2}}}-\frac{4 G^{2}\left(\frac{c_{0}}{(K+1)^{3}}+\frac{\hbar^{2} \kappa}{K+1}\right)}{\hbar^{2}\left(1+\frac{4 G^{2}}{\hbar^{2}(K+1)^{2}}\right)^{2}}\right) .
$$

As long as the "interacting" principal quantum number, $(K+1+\Delta l)$, in eq. (26) can be at most close to integer, the degeneracy of states within the $(K / 2, K / 2)$ multiplet in eq. (99) is relaxed. Nonetheless, as visible from eq. (27) the spectrum is still patterned after the $S O(4)$ levels $\mid K l m>$, and it still falls into an $\infty$ d unitary representation of the conformal group. As we shall see below, the $\Delta l$ contributions will have a detectable effect only on the masses of states with $l=0$ and will be helpful in removing the degeneracy between $P_{1 / 2}-S_{1 / 2}$ states, while leaving all the other excitations practically degenerate. The latter property of the interacting conformal rigid rotor parallels on $S^{3}$ the kinetic fine level splittings of a hydrogenic two-body system in a plane space and will occasionally be referred to as "curved" fine level splitting.

In effect, the relativistic framework of the conformal scale equation (7) on $\mathbf{R}^{1} \otimes S^{3}$, gauged (18), provides the intriguing possibility of having exclusively bound states organized into conformal bands whose states are not necessarily perfectly degenerate though they still keep spreading around $S O(4)$ levels. The case in which one of the particles is a spin- $1 / 2$ fermion is easily incorporated into the formalism by coupling the Dirac spinor $(1 / 2,0) \oplus(0,1 / 2)$ to $\mid K l m>$ in eq. (9). The excitations of such a system populate an infinite staircase (that is 
an $\infty$ d unitary $\mathrm{SO}(2,4)$ representation) whose ladders are given by reducible $\mathrm{SO}(4)$ representations as,

$$
\left(\frac{K}{2}, \frac{K}{2}\right) \otimes\left[\left(\frac{1}{2}, 0\right) \oplus\left(0, \frac{1}{2}\right)\right], \quad K=0,1,2, . . \infty
$$

Such multiplets consist of $K$ parity dyads of rising spins, $j^{P}=\frac{1}{2}^{ \pm}, \ldots,\left(K-\frac{1}{2}\right)^{ \pm}$, and a single-parity state of maximal spin, $j^{P}=\left(K+\frac{1}{2}\right)^{P}$. The absolute value of the parity of the two-body system depends on the parity of the scalar body, $\pi$, and is given by $P=\pi(-1)^{l}$. Alternatively, one also could have started from the very beginning with the non-relativistic stationary Schrödinger equation on $S^{3}$ with the $\cot \chi$ potential as

$$
\left[\frac{\hbar^{2}}{2 m} \kappa \mathcal{K}^{2}-2 G \kappa \cot \chi-\mathcal{E}\right] \psi(\chi)=0,
$$

an option first considered by Schrödinger [32].

This option has been investigated by us in our prior works [34], [35]. In contrast to eq. (18), the linear in mass Schrödinger equation (31) keeps respecting in the interacting case the degeneracies of the free geodesic motion [44]. In the present work we systematically departure from eq. (18). We shall compare outcomes of these two schemes in due places.

\section{QUARK-DIQUARK MODEL ON $S^{3}$.}

From now onward we assume dominance of quark-diquark configurations in the internal nucleon and $\Delta$ structures and apply eq. (18) to the description of the relative motion of these two bodies. We moreover shall consider the diquark as spin-less, a restriction that enables one to hit a specifically simple unitary $S O(2,4)$ representation. As we shall see below, this configuration turns out to be the quite adequate for data description. In considering the diquark as spinless, the total spin, $J$, of the resonance is then obtained through coupling the spin of the quark to the $q-(q q)$ relative angular momentum, $l$. For the time being, and because of the absence of spin-flavor interactions in the wave equation under consideration, we shall factorize the light flavor quantum number (actually isospin). In due course we shall see that the spectra reported so far do not contradict the above assumptions. The application of the conformal equation (7) to such two-body systems is then straightforward and can be featured as follows: 
- The relative angular momentum between the quark and the spin-less diquark takes the values $l \in[0, K]$, in accord with the conformal branching rule connecting the $\mathcal{K}^{2}$, and $\mathbf{L}^{2}$ eigenvalues in eq. (9).

- The total spin, $J$, of the $q-(q q)$ system is obtained from coupling the quark spin to that very $l$ as

$$
\left[\vec{l} \otimes \frac{\overrightarrow{1}}{2}\right]^{J M}, \quad J=l \pm \frac{1}{2}
$$

- In effect, one finds the following fermionic $S O(4)$ multiplets,

$$
J \in\left(\frac{K}{2}, \frac{K}{2}\right) \otimes\left[\left(\frac{1}{2}, 0\right) \oplus\left(0, \frac{1}{2}\right)\right], \quad l \in[0, K],
$$

emerging as levels of a conformal band corresponding to,

$$
K \in[0, \infty)
$$

Accordingly, the total spin $J$ in eq. (32) takes the following values,

$$
J=\frac{1}{2},\left(1 \pm \frac{1}{2}\right),\left(2 \pm \frac{1}{2}\right), \ldots,\left(K \pm \frac{1}{2}\right),
$$

to be identified with the spins of the light flavor baryon resonances.

- The parity, $P$, of the resonances, $P=\pi(-1)^{l}$, is the product of the parity, $\pi= \pm$, of the spinless diquark, and $(-1)^{l}$, the parity of the relative angular motion. The parity of the diquark, scalar or pseudoscalar, is fixed by matching parity, $P_{\max }$, of the highest spin, $J=K+\frac{1}{2}$, i.e.

$$
\text { If } J^{P}=\left(K+\frac{1}{2}\right)^{P_{\max }}, \quad \text { then } \pi=P_{\max }(-1)^{K} .
$$

- As a working hypothesis, to be tested by comparison with data, the nucleon diquark has been set as a scalar for the ground state and the low lying excitations with masses below $1600 \mathrm{MeV}$, and as a pseudo-scalar above.

- The $\Delta$ diquark has been set as an axial vector in the ground state and as a pseudoscalar above.

- The pseudo-scalar diquark is a $P$ wave axial-vector whose total angular momentum equals zero, and emerges from coupling the axial spin vector, $\vec{S}=\overrightarrow{1}^{+}$, to an internal vectorial, $1^{-}$, excitation according to $\left[\overrightarrow{1}^{+} \otimes \overrightarrow{1}^{-}\right]^{0^{-}}$. 
- The quark-diquark system is described by means of eq. (22) with the wave functions in eq. (25) . Insertion of the $\chi$ parametrization from eq. (5) into eq. (22) amounts to the following spatial $q-(q q)$ wave function,

$$
\Psi_{K l}(\chi(r))=N_{K l} e^{-a \sin ^{-1} r \sqrt{\kappa}}(r \sqrt{\kappa})^{K+1+\Delta l} R_{K-l}^{(a, b)}\left(\cot \sin ^{-1} r \sqrt{\kappa}\right) .
$$

It represents the radial dependence of the total wave function within the finite volume position space,

$$
r \sqrt{\kappa} \in[0,1] .
$$

- The energies $E$ in eq. (26), will be subsequently re-denoted by $M$ and given interpretation of resonance masses read off from the respective ground state $N$ and $\Delta$ masses.

In this fashion, the spatial part of the total baryon wave function has been designed to account for conformal symmetry in accord with $\mathrm{AdS}_{5} / \mathrm{CFT}_{4}$ on the one side, and with the (approximate) conformal symmetry of the QCD Lagrangian in the light flavor sector, on the other. In addition, $\Psi_{K l}(\chi(r))$ describes a confinement of finite range. In the next section we shall compare the outcome of such a model with data on $N$ and $\Delta$ resonances.

\section{A. The $\mathbf{N}$ and $\Delta$ spectra}

The spectrum of the nucleon continues being under debate despite the long history of the respective studies [1], [2]. Yet, unprejudiced inspection of the data reported by the Particle Data Group [4] reveals systematic and hardly to overlook grouping of the excited states of the baryons of the best coverage, the nucleon, and the $\Delta(1232)$. Take as a prominent example the seven $\Delta$ resonances $S_{31}(1900), P_{31}(1910), P_{33}(1920), D_{33}(1940), F_{35}(1905), D_{35}(1930)$, and $F_{37}(1950)$, which are squeezed in the narrow mass band between $1900 \mathrm{MeV}$ to 1950 $\mathrm{MeV}$ and which, given the limitations of data accuracies, are practically mass degenerate. This sequence of resonances consists of 3 parity dyads with spins ranging from $\frac{1}{2}^{ \pm}$to $\frac{5}{2}^{ \pm}$ and of a single parity state of maximal spin, $J^{\pi}=\frac{7}{2}^{+}$and its quantum numbers fit into the $K=3$ multiplet in eqs. (33), (35) . In the nucleon spectrum, one finds the $N(1440)-N(1535)-$ $N(1520)$ triplet, which would match quantum numbers of $K=1$ in eq. (33)). Patterns of similar type do not restrict to these two examples alone but extend to the spectrum of any of the lightest flavor baryons, no matter $N$, or $\Delta$. Notice the following observations. 
- The $\Delta(1232)$ excitations around $1700 \mathrm{MeV}$ form a group of three that contains one parity dyad with lowest spin $\frac{1}{2}^{ \pm}$and one single parity state of maximal spin, $J^{\pi}=\frac{3}{2}^{-}$ and would match $K=1$ in eq. (33),

- The $\Delta$ resonances around $1900 \mathrm{MeV}$ are just the $K=3$ example from above, which consisted of the three mass-degenerate parity dyads with spins ranging form $\frac{1}{2}^{ \pm}$to $\frac{5}{2}^{ \pm}$, and the one unpaired state of maximal spin, $\frac{7}{2}^{+}$, of practically same mass.

- Another spin-sequence could match $K=5$ in eq. (33) and is well marked by the parity simplex $J^{\pi}=\frac{11}{2}^{+}$of maximal spin in this region, the four star resonance $H_{3,11}(2420)$. The pairs $H_{39}(2300)-G_{39}(2400)$, and $G_{37}(2200)-F_{37}(2390)$ would provide two of the five parity dyads necessary to complete $K=5$ in eq. (33). The $D_{35}(2350)$ would be suited as a part of a $\frac{5}{2}^{ \pm}$doublet, while spin $\frac{1}{2}^{ \pm}$and $\frac{3}{2}^{ \pm}$pairs are "missing".

It verifies directly by inspection that the mass-splittings inside the above spin-cascades are notably smaller than the splittings between the averaged cascade masses. In this fashion the $\Delta$ spectrum reveals a level structure. The $N$ excitations follow quite same patterns though appear shifted downward in mass by about $200 \mathrm{MeV}$ relative to the $\Delta$ excitations. The attention to this clustering phenomenon in light baryon spectra has been drawn first in ref. [45] and was further elaborated in refs. [46]-[48]. In assuming equality of the quantum numbers of the $N$ and $\Delta$ excitations and comparing both spectra, allows to pin down the states missing for the completeness of the scheme.

Under the assumption of $q-(q q)^{0^{ \pm}}$as a dominant configuration of internal baryon structure, the above clustering phenomenon is shown below to find a quantitative explanation in terms of the levels of the spectrum of eqs. (22), (26), (33), and (35), and is interpreted here as a hint on possible realization of conformal symmetry in the light flavor baryon spectra.

$\underline{\text { Missing resonance predictions. }}$

The comparison of the spectrum in eq. (26) to data [4] is presented in Figs. 1, and 2. We predict one $P_{31}$ "missing" state from the $K=1$ level. The three more states, $S_{31}, P_{33}$, and $D_{35}$ are needed for the completeness of the $K=2$ level. The $K=3$ level is complete. From the required nine states in the next $K=4$ level, $S_{31}$, and $G_{37}$, have been in turn identified with the observed one star resonances $\Delta(2150)$, and $\Delta(2200)$. Our $F_{35}$ has been 


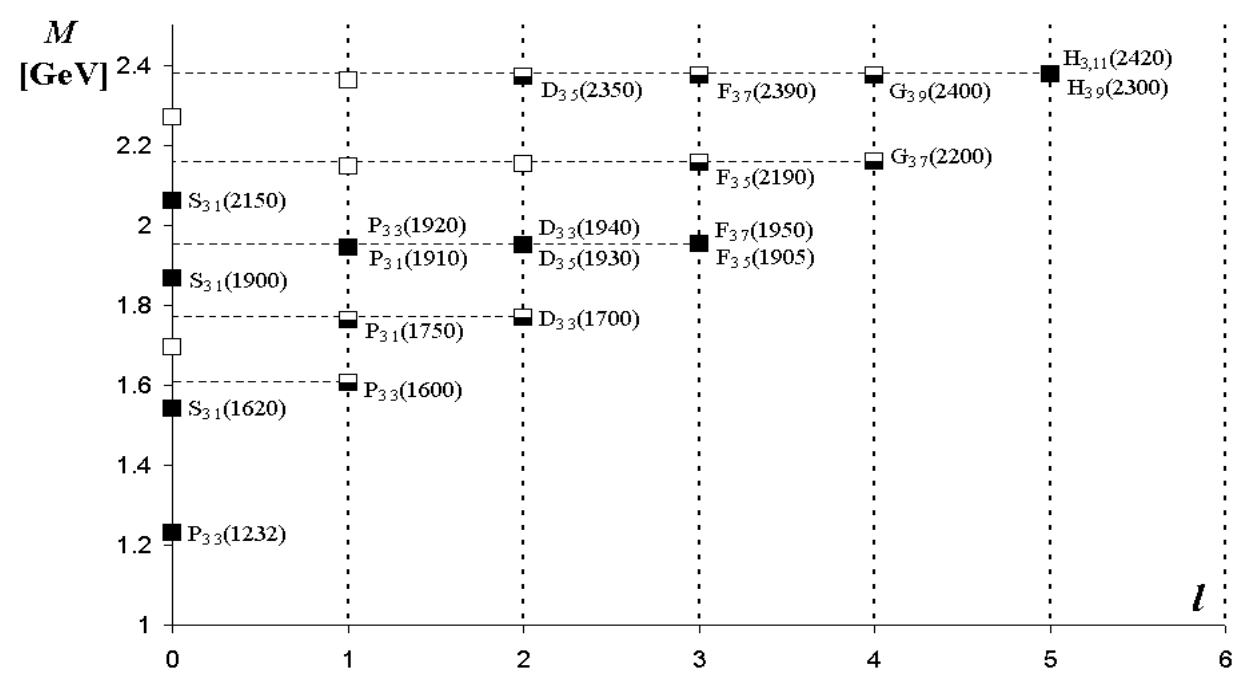

FIG. 1: Assignments on the $l / M$ grid of the reported [4] $\Delta$ excitations to the $\mathbf{R}^{1} \otimes S^{3}$ spectrum in eq. (26) . The distribution of the reported (exp) resonances over the predicted (th) states has been obtained from a least mean square four parameter data fit, i.e. by minimizing $\sigma=$ $\sqrt{\frac{1}{N} \sum_{i=1}^{i=N}\left(M_{\mathrm{th}}^{(i)}-M_{\mathrm{exp}}^{(i)}\right)^{2}}$. The sum includes all the reported resonances. The minimal value, $\sigma_{\min }=0.043 \mathrm{GeV}$ has been obtained for the following potential parameters: $G=0.04933 \mathrm{GeV} \cdot \mathrm{fm}$, $R=0.747 \mathrm{fm}, \bar{a}=0.5037 \mathrm{GeV}$, and $\mu=1.044 \mathrm{GeV}$. The excitations, $E$, set equal to masses, have been read off from the $\Delta(1232)$ mass. Full and empty squares denote reported and predicted states, respectively.

associated with the two-star resonance $\Delta(2000)$, for which the two quite different mass values of $(1752 \pm 32)$, and $(2200 \pm 125)$ have been listed in [4]. We here adopt the position of ref. [10] and consider this resonance as $\Delta(2190)$, which allows to place it well in the $K=4$ multiplet. The remaining six states from that very level are missing (see Table I.) Finally, the $K=5$ members $H_{39}$, and $G_{39}$ can be assigned to the respective two-star resonances $\Delta(2300)$, and $\Delta(2400)$, while $\Delta(2350)$ and $\Delta(2390)$ are good candidates for $D_{35}$, and $F_{37}$. This level is well marked by its highest spin $H_{3,11}$, the four-star resonance $\Delta(2420)$. We find 16 resonances missing from the first five levels of the the conformal band covering the $\Delta$ spectrum. On the nucleon side the highest spins , $F_{17}$ from the $K=3$, and $H_{31,11}$ from $K=5$ are "missing" . The $K=2$ level is completely "missing". Compared to our previous work [34],[35], the fit places the $\mathrm{N}(1900), \mathrm{N}(1990)$, and $\mathrm{N}(2000)$ resonances at the lower $K=4$ level and leaves instead $P_{11}, F_{15}$, and $F_{17}$ in $\mathrm{K}=5$ unoccupied. The number of "missing" nucleonic states 


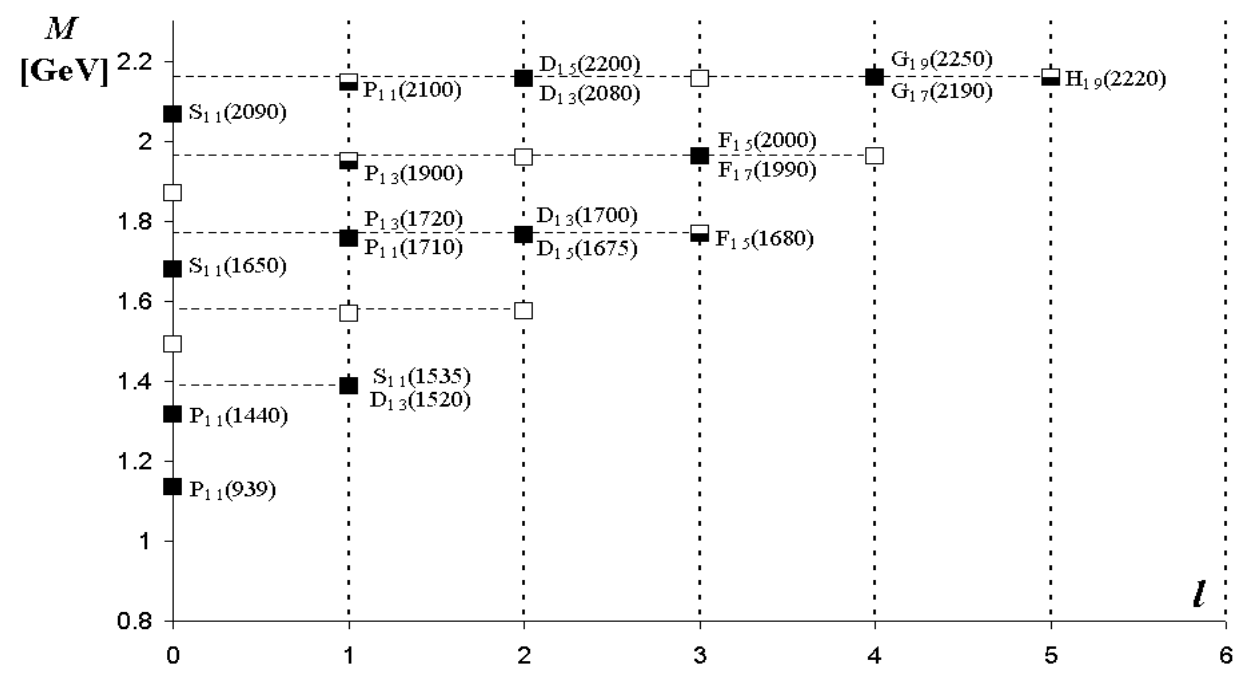

FIG. 2: Assignments on the $l / M$ grid of the reported [4] $N$ excitations to the $\mathbf{R}^{1} \otimes S^{3}$ spectrum in eq. (26). The distribution of the reported (exp) resonances over the predicted (th) states has been obtained from a four parameter least mean square data fit, i.e. by minimizing $\sigma=$ $\sqrt{\frac{1}{N} \sum_{i=1}^{i=N}\left(M_{\mathrm{th}}^{(i)}-M_{\mathrm{exp}}^{(i)}\right)^{2}}$. The sum includes all the reported resonances. The value of the proton mean square charge radius has been also taken into consideration by the fit. The minimal value of $\sigma_{\min }=0.0855 \mathrm{GeV}$ has been obtained for the following potential parameters: $G=0.0493$ $\mathrm{GeV} \cdot \mathrm{fm}, R=0.9814 \mathrm{fm}$, and $\mu=0.3213 \mathrm{GeV}$, and $\bar{a}=0.932 \mathrm{GeV}$. The excitations, $E$, set equal to masses, have been read off from the nucleon mass. Other notations as in Fig. 1

is sixteen (see Table I). Therefore, for baryons whose internal structure is dominated by the $q-(q q)^{0^{ \pm}}$configuration, we predict a total of 32 nucleon and $\Delta$ resonances "missing" from the first five levels of the respective nucleon, and $\Delta$ conformal bands. Figures and tables show that the $N$ and $\Delta$ resonances reported so far are pretty well matched by the excitations of this simplest configuration and are illustrative of a well pronounced footprint of conformal symmetry in the spectra of the lightest flavor baryons. In case the diquarks were to carry higher angular momenta, the excitations of $q-(q q)^{l}$ with $l>0$ would populate higher $S O(2,4)$ representations, which one can expect to appear much heavier in mass. One of the conclusions following from our findings is that at most "missing" states belonging to different levels of the conformal band might still have chances to be pinned down by an $O(3)$ partial wave analysis. In contrast, the states belonging to same level, in being strongly overlapping, are better looked up in terms of $O(4)$ partial wave analysis and identified as a 
whole.

In conclusion, the confirmation by data of the predicted degeneracy among the parity pairs belonging to same $K$ and displayed in Figs. 1 and 2, signals relevance of conformal symmetry for the spectra of the light flavor baryons.

Before proceeding further, a comment is in order on the degeneracies predicted by the LightFront QCD framework [8], [9]. There, one finds the mass formula as

$$
M^{2}=N, \quad N=1,2,3, \ldots, \quad N=n+\nu+1,
$$

and observes again a conformal band (as it should be) with respect to $\nu$. The relation of the light-cone variable $\nu$ to ordinary angular momentum is more involved. It equals $\nu=L$ for mesons, and $\nu=L+1$ for baryons. Also the Light-Front QCD formalism reports degeneracies of excited $N$ and $\Delta$ states, that partly overlap with those considered here. Examples are the positive parity spin-sequences $\mathrm{F}_{37}(1950)-\mathrm{F}_{35}(1905)-\mathrm{P}_{33}(1920)-\mathrm{P}_{31}(1910)$ from the $K=3$ level in Fig. 1, and the $\mathrm{F}_{15}(1680)-\mathrm{P}_{13}(1720)$ states from same level in the nucleon spectrum in Fig. 2. So far, the question on the degeneracies among opposite parities has not been addressed in refs. [8], [9].

$\underline{\text { Mass ordering in } P_{2 I, 1}-S_{2 I, 1} \text { pairs. }}$

The model predicts the correct mass ordering of the $P_{11}-S_{11}$ states through the spectrum. Within the framework of the present study the numerical value of the splittings between such states is entirely determined by the gauged centrifugal barrier, $\bar{U}_{l}(\chi, \kappa)$, defined in eqs. (22), which prescribes that $l=1$ states will appear higher in mass than those with $l=0$. The ordering, $P_{2 I, 1}-S_{2 I, 1}$ versus $S_{2 I, 1}-P_{2 I, 1}$ depends on the parity of the diquark. When the diquark is a scalar, spin- $\frac{1}{2}^{+}$and spin- $\frac{1}{2}^{-}$in turn refer to zero and unit underlying angular momenta and are associated with $P_{2 I, 1}$, and $S_{2 I, 1}$ states. This is the reason for which at the scale of $1500 \mathrm{MeV}$, where the diquark is a scalar, the measured $P_{11}(1440)$ state appears lower in mass than its $S_{11}(1535)$ neighbor. From the $1700 \mathrm{MeV}$ level onward, the parity of the diquark changes to pseudoscalar, and it is $S_{11}$ that has zero angular momentum, in accord to eq. (36). Consequently, $S_{11}$ states with masses above $1600 \mathrm{MeV}$ appear systematically at lower masses than their nearest $P_{11}$ neighbors. Examples are the $S_{11}(1650)-P_{11}(1710)$, 
and $S_{11}(2090)-P_{11}(2100)$ pairs. Recall that originally the suggestion on the parity change of the diquark was made with the purpose of matching parity of the highest spins in the fermionic multiplets in eqs. (35), (36). Therefore,

the reverse mass ordering in the $S_{11}-P_{11}$ pairs above $1600 \mathrm{MeV}$ relative to the $P_{11}(1440)-S_{11}(1535)$ splitting, provides an independent argument in favor of the change of parity of the diquark from scalar to pseudoscalar at that scale.

In the $\Delta$ spectrum, where the diquark was fixed to a pseudoscalar for all the excited states, we find similar $S_{31}-P_{31}$ splittings. The mass ordering in the $S_{31}(1900)-P_{31}(1910)$ pair, also correctly described within the model presented, serves as an example. As to the resonances with $l>1$, the $\Delta l$ corrections to $l$ in eq. (21) become insignificant and the splittings practically disappear (see Tables I and II).

In conclusion, we observed that the quantum numbers of the reported nucleon resonances are close to equal to those of the reported $\Delta$ resonances (compare Tables $\mathbb{I}$, and II). We took advantage of this circumstance to embed the reported states isospin by isospin into conformal bands of the type in eq. (33). In so doing we patterned the $N$, and the $\Delta$ spectrum each after an unitary representation of the conformal group. So far, no reported state drops out of the suggested systematics. We predicted a total of 32 "missing" resonances needed for the completeness of the conformal nucleon and $\Delta$ bands.

\section{B. Charge radii and form factors}

The effect of curvature on the physical observables is two-fold. On the one side, it encodes the topology of the position space and will influence Fourier transforms through the $S^{3}$ integration volume,

$$
\mathrm{d} \Omega_{3}=\sin ^{2} \chi \sin \theta \mathrm{d} \chi \mathrm{d} \theta \mathrm{d} \varphi=(r \sqrt{\kappa})^{2} \sin \theta \mathrm{d}\left(\sin ^{-1} r \sqrt{\kappa}\right) \mathrm{d} \theta \mathrm{d} \varphi
$$

where use has been made from the parametrization in eq. (15). The curved integration

volume, $(r \sqrt{\kappa})^{2} d \sin ^{-1} r \sqrt{\kappa}$, approaches the flat one, $\kappa^{\frac{3}{2}} r^{2} d r$, only in the small $\chi$ angle approximation. On the other side, curvature can be viewed as a new phenomenological potential parameter. The first aspect is of crucial importance in performing the Fourier 
TABLE I: Predicted excitation values of the $\Delta$ states in the $K=0 \div 5$ levels of the conformal band in eq. (9) calculated according to eq. (26). The internal angular momenta take the values, $l=0,1,2, . . K$. "Missing" states are marked by boldface. The number in the parenthesis gives the predicted mass of excitations [in $\mathrm{MeV}]$ carrying same internal angular momentum. States with $J=l \pm \frac{1}{2}$ appear degenerate within the present scheme because of the absence of spinorbit interactions in the present stage model. Notice that differently from its excitations, $\Delta(1232)$ belongs to $\left(\frac{3}{2}, 0\right) \oplus\left(0, \frac{3}{2}\right)$.
$\mathrm{K} \quad \mathrm{l}=0$
$l=1$
$l=2$
$l=3$
$l=4$
$l=5$

$\mathrm{K}=0 \mathrm{P}_{31}(1230)$

$\mathrm{K}=1 \mathrm{~S}_{31}(1542) \quad \mathbf{P}_{31} / \mathrm{P}_{33}(1607)$

$\mathrm{K}=2 \mathbf{S}_{\mathbf{3 1}}(1699) \quad \mathrm{P}_{31} / \mathbf{P}_{33}(1768) \quad \mathrm{D}_{33} / \mathbf{D}_{\mathbf{3 5}}(1774)$

$\mathrm{K}=3 \mathrm{~S}_{31}(1874) \quad \mathrm{P}_{31} / \mathrm{P}_{33}(1953) \quad \mathrm{D}_{33} / \mathrm{D}_{35}(1960) \quad \mathrm{F}_{35} / \mathrm{F}_{37}(1963)$

$\mathrm{K}=4 \mathrm{~S}_{31}(2072) \quad \mathbf{P}_{\mathbf{3 1}} / \mathbf{P}_{\mathbf{3} 3}(2159) \mathbf{D}_{\mathbf{3} 3} / \mathbf{D}_{\mathbf{3 5}}(2167) \mathrm{F}_{35} / \mathbf{F}_{\mathbf{3} 7}(2170) \mathrm{G}_{37} / \mathbf{G}_{\mathbf{3 9}}(2171)$

$\mathrm{K}=5 \mathbf{S}_{\mathbf{3 1}}(2287) \quad \mathbf{P}_{\mathbf{3 1}} / \mathbf{P}_{\mathbf{3} 3}(2380) \mathbf{D}_{\mathbf{3} 3} / \mathrm{D}_{35}(2388) \mathbf{F}_{\mathbf{3 5}} / \mathrm{F}_{37}(2391) \mathbf{G}_{\mathbf{3} 7} / \mathrm{G}_{39}(2393) \mathrm{H}_{39} / \mathrm{H}_{3,11}(2394)$

transform of the cotangent potential and constructing an effective instantaneous gluon propagator, in parallel to the instantaneous photon propagator obtained from Fourier transforming the Coulomb potential. Exploring this aspect is the subject of section 4 . Compared to this, calculations of form-factors of states of low internal angular momentum, such as the ground state, and the $P_{11}(1440)$ resonance, are much less affected by the 
TABLE II: Predicted excitation values of the nucleon states in the $K=0 \div 5$ levels of the conformal band in eq. (9) calculated in accord with eq. (26). Other notations as in Table 1.

\begin{tabular}{|c|c|c|c|c|c|}
\hline $\mathrm{K} \quad \mathrm{l}=0$ & $l=1$ & $l=2$ & $l=3$ & $l=4$ & $l=5$ \\
\hline $\mathrm{K}=0 \quad \mathrm{P}_{11}(1136)$ & - & - & - & - & - \\
\hline $\mathrm{K}=1 \mathrm{P}_{11}(1316)$ & $\mathrm{S}_{11} / \mathrm{D}_{13}(1387)$ & - & - & - & \\
\hline $\mathrm{K}=2 \mathbf{P}_{\mathbf{1 1}}(1492)$ & $\mathbf{S}_{11} / \mathbf{D}_{13}(1568)$ & $\mathbf{P}_{\mathbf{1 3}} / \mathbf{F}_{\mathbf{1 5}}(1574)$ & - & - & - \\
\hline$K=3 S_{11}(1678)$ & $\mathrm{P}_{11} / \mathrm{P}_{13}(1757)$ & $\mathrm{D}_{13} / \mathrm{D}_{15}(1764)$ & $\mathrm{F}_{15} / \mathbf{F}_{\mathbf{1 7}}(1767)$ & - & - \\
\hline $\mathrm{K}=4 \mathbf{S}_{11}(1870)$ & $\mathbf{P}_{11} / \mathrm{P}_{13}(1951)$ & $\mathbf{D}_{13} / \mathbf{D}_{15}(1958)$ & $\mathrm{F}_{15} / \mathrm{F}_{17}(1961)$ & $\mathbf{G}_{\mathbf{1 7}} / \mathbf{G}_{\mathbf{1 9}}(1962)$ & - \\
\hline $\mathrm{K}=5 \mathrm{~S}_{11}(2066)$ & $\mathrm{P}_{11} / \mathbf{P}_{\mathbf{1 3}}(2147)$ & $\mathrm{D}_{13} / \mathrm{D}_{15}(2154)$ & $\mathbf{F}_{\mathbf{1 5}} / \mathbf{F}_{\mathbf{1 7}}(2157)$ & $\mathrm{G}_{17} / \mathrm{G}_{1,9}(2159)$ & $\mathrm{H}_{1,9} / \mathbf{H}_{\mathbf{1}, 11}(2160)$ \\
\hline
\end{tabular}

integration volume, because the wave functions practically correspond to the small $\chi$ angle approximation, and thereby approach flat-space wave functions. In these calculations the importance of curvature is more to provide a phenomenological parameter in addition to the potential strength and facilitate data fits.

The standard scheme for calculating form-factors relies upon Fourier transforms from position to the conjugate momentum space by means of the $3 \mathrm{~d}$ plane wave, exp $i \mathbf{q} \cdot \mathbf{r}$. This plane wave can be regarded as the special case of the $\mathbf{R}^{4}$ plane wave, $\exp \left(i q_{4} x_{4}+\mathbf{q} \cdot \mathbf{r}\right)$, in which $q_{4}=0$. From this perspective, the $3 \mathrm{~d}$ plane wave that accounts for a position vector 
$\mathbf{r}$ restricted to the equatorial plane of $S^{3}$ reads,

$$
\left.e^{i q_{4}+i|\mathbf{q}||\mathbf{r}| \cos \theta}\right|_{q_{4}=0}=e^{i|\mathbf{q}||\mathbf{r}| \cos \theta}=e^{i|\mathbf{q}| \frac{\sin \chi}{\sqrt{\kappa}} \cos \theta}, \quad|\mathbf{r}|=R \sin \chi=\frac{\sin \chi}{\sqrt{\kappa}}, \quad r \sqrt{\kappa} \in[0,1],
$$

and refers to a $z$ axis chosen along the momentum vector (a choice justified in elastic scattering).

Electric charge form-factors are the simplest physical observables to calculate, and the corner stone of any spectroscopic model. They reduce to the Fourier transform of the charge density, proportional to $\left|\Psi_{K l}(\chi)\right|^{2}$ in our case. The extraction of the mean square charge radius, $\left\langle\mathbf{r}^{2}\right\rangle$, from the form-factor is then standard and calculated as the slope at origin. We here choose as illustrative examples the mean square charge radii of the proton, the $P_{11}(1440)$ and $S_{11}(1535)$ resonances. The proton ground state wave function entering the calculation is obtained from eq. (25), the explicit expression for the normalization factor being

$$
N_{(00)}=\frac{4 b\left(a^{2}+1\right)}{1-e^{-2 \pi a}}, \quad a=\frac{2 G E_{0}}{\hbar^{2} \sqrt{\kappa}\left(1-\frac{1}{2}+\sqrt{\frac{1}{4}-\frac{(2 G)^{2}}{\hbar^{2}}}\right)}, \quad E_{0}=\bar{a}+\sqrt{\frac{1}{2} \mu^{2}-\frac{\hbar}{4}} \kappa
$$

The mean square charge radius for this state expresses in closed form as,

$$
<\mathbf{r}^{2}>_{p}=\frac{6}{\left(4 a^{2}+9\right) \sqrt{\kappa}} .
$$

With the potential parameters fitted to the spectra, we find,

$$
\sqrt{\left.<\mathbf{r}^{2}\right\rangle_{p}}=0.664 \mathrm{fm}
$$

to be compared to the experimental value of $\sqrt{\left\langle\mathbf{r}^{2}\right\rangle_{p}}=0.8750 \pm 0.008 \mathrm{fm}$ reported by [4]. Comparison of the predicted to the measured proton electric charge form factor is presented in Fig. 3. For the Roper resonance, same observable is calculated as

$$
<\mathbf{r}^{2}>_{\text {Roper }}=\frac{2\left(5+52 a^{2}\right)}{\left(10 a^{4}+104 a^{2}+25\right) \kappa}, \quad a^{2}=\frac{4 G^{2}(E-\bar{a})^{2}}{\hbar^{4} \kappa(\alpha(l)+1)^{2}} .
$$

The resulting value is

$$
\sqrt{<\mathbf{r}^{2}>_{\text {Roper }}}=0.8484 \mathrm{fm}
$$

As to the $S_{11}(1535)$ resonances, the prediction is numerical and obtained as

$$
\sqrt{<\mathrm{r}^{2}>\mathrm{S}_{11}}=0.8754 \mathrm{fm}
$$




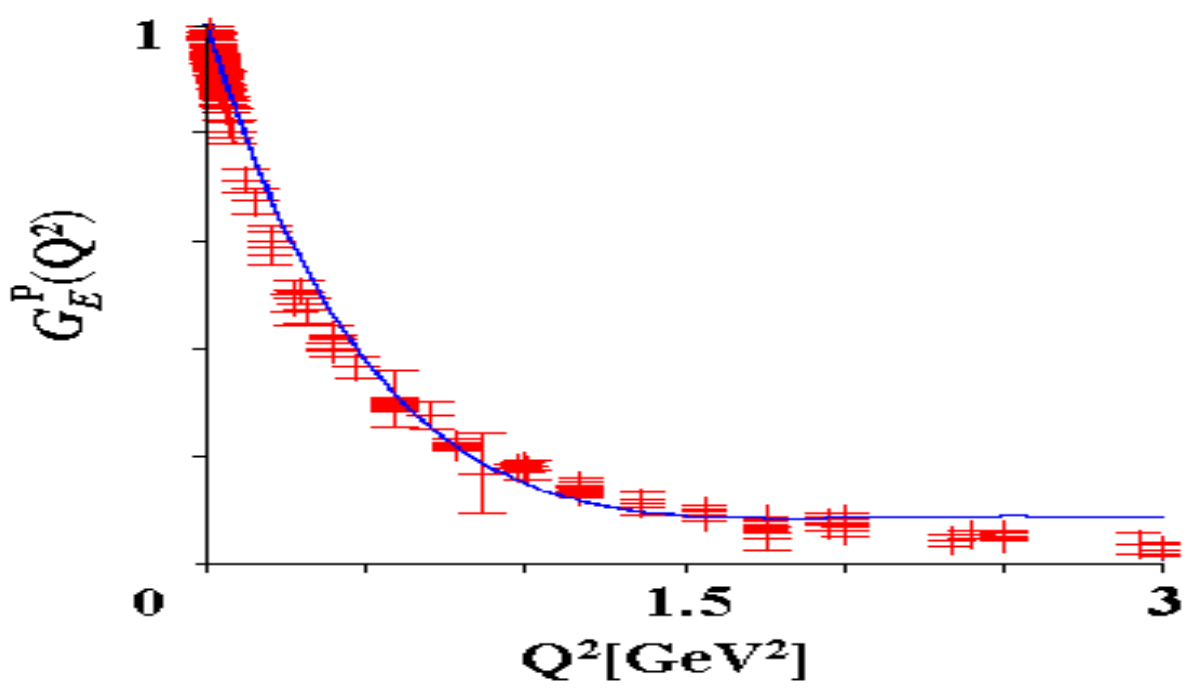

FIG. 3: The proton electric charge form factor in comparison to data. The solid line refers to the present calculation in terms of the solutions to eq. (22). The potential parameters are given in the caption of Fig. 2, Data compilation same as in ref. [49].

The proton form factor is compared to data in Fig. 3. The comparison of the other two form-factors to that of the proton is presented in Fig. 4. We obtain the mean square charge radius of the proton-like Roper resonance enhanced by a bit less than $30 \%$ over the proton charge radius. Nonetheless, compared to the proton, the form factor of the $P_{11}(1440)$ takes smaller values. This because our predicted $P_{11}(1440)$ charge density appears slightly arced at origin.

An enhancement, though smaller (10\%) has been found by Nagata and Hosaka in ref. [50]. One of the differences between the model by Nagata-Hosaka and the present model is that while in the former both the scalar and axial vector diquarks are weighted by non-zero form factors, we here weight them by the extremal 1 and zero values, respectively. This for the sake of staying as close to conformal symmetry as possible. In the present model, it is the curvature parameter that seems to account for some of the effects governed by the diquark form factors in flat space quark models. Curvature as a phenomenological tool suited in simulating complicated many-body effects is known to be useful in the description of such complicated many-body problems as Brownian motion, plasma correlations, instanton physics etc. [51], [12]. A reason for which the replacement of the complicated many-body problem of baryon structure (the genuine baryon wave function contains next to q-(qq) also 


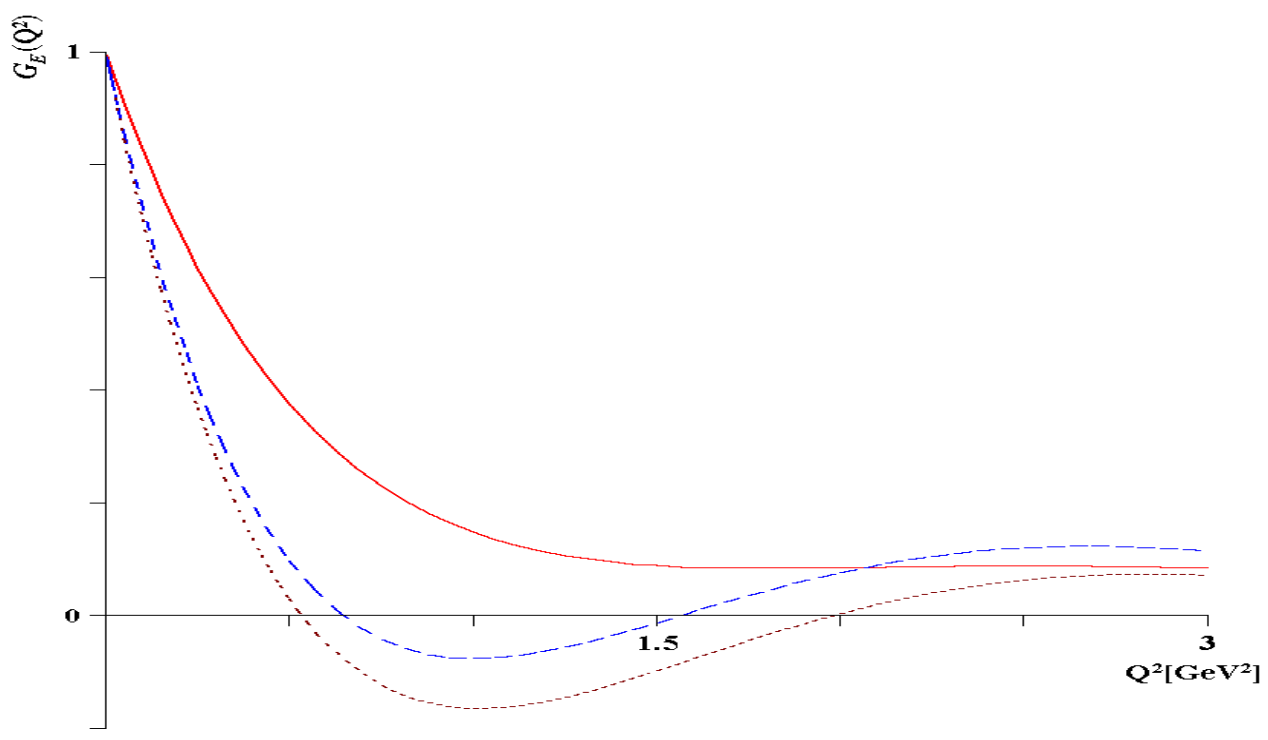

FIG. 4: The electric charge form factors of the $P_{11}(1440)$, and $S_{11}(1650)$ resonances (dashed and dotted lines, respectively), in comparison to the proton electric charge form factor (solid line).

$3 \mathrm{q}, 3 \mathrm{q}(\bar{q} \mathrm{q})^{n}, 3 \mathrm{q}\left(\mathrm{g}^{n}\right)$ etc configurations) by the simple q-qq problem on $S^{3}$ turns out to be a useful approximation to reality is that curvature, in combination with the conformal gauge potential, reasonably accounts for the omitted many body effects.

In the range of $Q^{2} \in[0,1.5) \mathrm{GeV}^{2}$, the proton form-factor of the present relativistic treatment compares in quality with $G_{E}^{p}\left(Q^{2}\right)$ reported earlier by us in ref. [35], where we employed a cot $+\csc ^{2}$ confinement potential in the stationary flat space radial Schrödinger equation, though the relativistic and non-relativistic charge density profiles, $\sim|\Psi|^{2}$, in the range of $\chi \in[0, \pi]$ are quite different (see Fig. 5 ). The profile of the proton charge density in the non-relativistic case is exclusively governed by the cotangent potential, while in the relativistic case it obtains significant contributions from both the gauge interaction and the gauged centrifugal barrier, $\bar{U}_{l}(\chi, \kappa)$ (defined in eqs. (21), (22) ). The cost for obtaining nonetheless similar $G_{E}^{p}\left(Q^{2}\right)$ form-factors in the above two distinct schemes has been admitting in the present data fit a larger least mean square error in comparison to the non-relativistic treatment of ref. [35]. Compared to $G_{E}^{p}\left(Q^{2}\right)$ obtained within the framework of relativistic quantum mechanics along the line of ref. [49] and referred to as GBE CQM there, our result on the mean square proton charge radius appears somewhat underestimated although our predicted $G_{E}^{p}\left(Q^{2}\right)$ values fall within the error bars of the reported data below $2 \mathrm{GeV}^{2}$, and lie somewhat above afterward. This satisfactory behavior of the proton electric charge form 


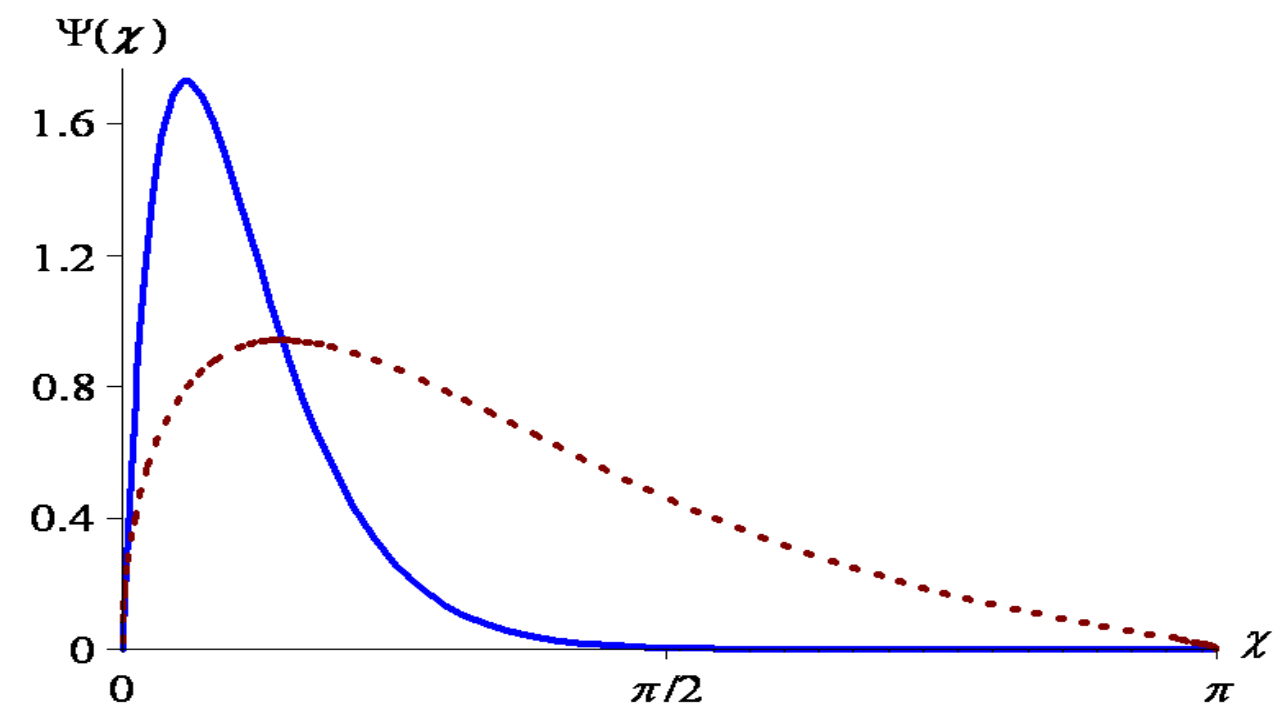

FIG. 5: Comparison of the Klein-Gordon (present) and Schrödinger [35] proton ground state wave functions (dashed and solid line, respectively).

factor is illustrative of the realistic character of the wave functions in eq. (37).

\section{DRESSING FUNCTION FOR THE GLUON PROPAGATOR IN THE IN- FRARED FROM FOURIER TRANSFORM OF THE $S^{3}$ POTENTIAL}

This section is devoted to an effective instantaneous gluon propagator constructed as a Fourier transform of the conformal gauge potential. This potential, a cotangent, captures quite well the essential traits of QCD dynamics in so far as it interpolates between the inverse distance potential (associated with the perturbative regime of the one gluon exchange) and the infinite well (associated with asymptotically free though trapped quarks) while passing through a region of linear growth (associated with the non-perturbative regime of flux-tube interactions), a result due to [35]. The finite range character of the gauge potential in eq. (17) is caused by the terms in its Taylor series decomposition that appear beyond the Coulombic+linear terms (as seen in eq. (16)), and which can be interpreted as phenomenological non-perturbative corrections to the former. For all these reasons, associating the Fourier transform of the cotangent function on $S^{3}$ with an effective gluon propagator seems justified. In the following we shall present this transformation and compare the outcome to Lattice QCD results. 
The gluon, $G_{\mu \nu}^{a b}\left(q^{2}\right)$, and ghost, $D^{a b}\left(q^{2}\right)$, propagators in the Landau gauge are defined in their turn as

$$
G_{\mu \nu}^{a b}=-i\left[\left(g_{\mu \nu}-\frac{q_{\mu} q_{\nu}}{q^{2}}\right) \frac{G\left(q^{2}\right)}{q^{2}}\right] \delta^{a b}
$$

and

$$
D^{a b}\left(q^{2}\right)=\frac{D\left(q^{2}\right)}{q^{2}} \delta^{a b}
$$

Here, $G\left(q^{2}\right)$ and $D\left(q^{2}\right)$ are referred to as the respective gluon and ghost dressing functions. The gluon propagator in this gauge is known to be transverse in the Lorenz indices, and $\Pi(q)$ stands for the gluon self energy. Gluon and ghost propagators can be obtained from solving Schwinger-Dyson equations. In so doing, especially simple expressions for $G\left(q^{2}\right)$ and $D\left(q^{2}\right)$ have been reported in [53] as

$$
G\left(q^{2}\right) \sim q^{2}, \quad D\left(q^{2}\right) \sim 1 / q^{4}
$$

meaning finiteness of the gluon propagator in the infrared. Contrary to this, the behavior of the ghost propagator is Coulombic in the infrared. Both propagators approach zero in the ultraviolet. More complicated expressions have been calculated in [54]. Independently, a finite gluon propagator in the infrared has also been calculated recently in lattice QCD [55].

In view of these properties of the gluon propagator, it is of interest to calculate the gluon dressing function from the $\mathbf{R}^{1} \otimes S^{3}$ quark model. To do so we apply the Born approximation to $E-V=\sqrt{\mathbf{p}^{2}+\mu^{2}}$ and calculate the instantaneous $\left(q_{0}=0\right)$ gluon propagator in parallel to our recent work [44] as a Fourier transform of the $\cot \chi(r)$ potential on $S^{3}$ employing the integration volume in eq. (40) in the parametrization of eq. (41).

In Cartesian coordinates the $\cot \chi(r)$ term equals $\frac{x_{4}}{r}$, and stands in fact for two potentials distinct by a sign and describing interactions on the respective Northern, and Southern hemispheres. Correspondingly, their respective Fourier transforms to momentum space become

$$
\begin{aligned}
4 \pi \Pi(|\mathbf{q}|)= & -2 \mu \frac{(-2 G \sqrt{\kappa})}{\hbar^{2}} \int_{0}^{\infty} d|x||x|^{3} \delta(|x|-R) \int_{0}^{2 \pi} d \varphi \int_{0}^{\pi} d \theta \sin \theta \\
& \int_{0 / \frac{\pi}{2}}^{\frac{\pi}{2} / \pi} d \chi \sin ^{2} \chi e^{i|\mathbf{q}| \frac{\sin \chi}{\sqrt{\kappa}} \mid \cos \theta} \cot \chi
\end{aligned}
$$

where the $\delta(|x|-R)$ function restricts $\mathbf{R}^{4}$ to $S^{3}$. It is the requirement on invertability of this transformation, addressed here for the first time, that demands for distinguishing between momentum space potentials on the Northern and Southern hemispheres of $S^{3}$. The first 
potential goes with $\chi \in\left[0, \frac{\pi}{2}\right]$, corresponds to a positive $x_{4}$, and describes an increasing $r \equiv|\mathbf{r}| \in[0, R]$. The second one refers to $\chi \in\left[\frac{\pi}{2}, \pi\right]$, a negative $x_{4}$, and describes a decreasing $|\mathbf{r}| \in[R, 0]$. To prove the invertability of the integral transform it is instructive to cast the transformation integral in eq. (51) to the following equivalent form,

$$
4 \pi \frac{\Pi(|\mathbf{q}|)}{\frac{(4 \mu G \sqrt{\kappa})}{\hbar^{2}}}= \pm \int_{0}^{R} \mathrm{~d} r \frac{r^{2}}{\sqrt{R^{2}-r^{2}}} \frac{\sqrt{R^{2}-r^{2}}}{r} \int_{0}^{2 \pi} d \varphi \int_{0}^{\pi} d \theta \sin \theta e^{i \mathbf{q} \cdot \mathbf{r}}= \pm 4 \pi \frac{1-\cos |\mathbf{q}| R}{\mathbf{q}^{2}}
$$

where we used the parametrization in eq. (41). Defining now the integral transform inverse to eq. (52) as,

$$
\begin{aligned}
& \pm \frac{\sqrt{R^{2}-r^{2}}}{(2 \pi)^{3}} \int_{0}^{\infty} \mathrm{d}|\mathbf{q}| \mathbf{q}^{2} \int_{0}^{2 \pi} \mathrm{d} \varphi \int_{0}^{\pi} \mathrm{d} \theta \sin \theta \frac{4 \pi(1-\cos |\mathbf{q}| R)}{\mathbf{q}^{2}} e^{-i \mathbf{q} \cdot \mathbf{r}} \\
& = \pm \frac{2}{\pi} \frac{\sqrt{R^{2}-r^{2}}}{r}\left\{\begin{aligned}
\frac{\pi}{2}, & & r<R, \\
\frac{\pi}{4}, & & r=R, \\
0, & r & >R,
\end{aligned}\right.
\end{aligned}
$$

proofs the invertability.

We here for concreteness pick up the Northern hemisphere potential and cast it in the more compact form,

$$
\Pi(|\mathbf{q}|)=c \frac{2 \sin ^{2} \frac{|\mathbf{q}|}{2 \hbar \sqrt{\kappa}}}{\left(\frac{|\mathbf{q}|}{\hbar \sqrt{\kappa}}\right)^{2}}, \quad c=\frac{4 G \mu}{\hbar^{2} \kappa} .
$$

It is increasing in the infrared, finite at origin, and approaches the Coulomb propagator in the ultraviolet. In the notations of eq. (48) our result takes the form

$$
\frac{G\left(\mathbf{q}^{2}\right)}{\mathbf{q}^{2}}=c \frac{2 \sin ^{2} \frac{|\mathbf{q}|}{2}}{\mathbf{q}^{2}}
$$

for a dimensionless q measured in units of $\hbar \sqrt{\kappa}$. Stated differently,

$$
G\left(\mathbf{q}^{2}\right)=2 c \sin ^{2} \frac{|\mathbf{q}|}{2}=c(1-\cos |\mathbf{q}|)=c\left(\frac{\mathbf{q}^{2}}{2 !}-\frac{\mathbf{q}^{4}}{4 !}+\frac{\mathbf{q}^{6}}{6 !}-\ldots\right),
$$

and in accord with eq. (50). Therefore, quark physics in $\mathbf{R}^{1} \otimes S^{3}$ also predicts a finite gluon dressing function in the infrared which approaches zero in the ultraviolet. Such a type of behavior has been observed, for example, in the description of confinement phenomena [55]. In summary, one of the virtues of the curvature aspect of the cotangent gauge potential is that its $S^{3}$ Fourier transform comes out well defined. 


\section{CONCLUSIONS}

In the present investigation we examined consequences of conformal symmetry in gravity-gauge duality on spectroscopic data on the lightest baryons, the nucleon and the $\Delta(1232)$. The $\mathrm{AdS}_{5} / \mathrm{CFT}_{4}$ concept on conformal symmetry has been implemented by a quark-diquark model placed directly on a conformally compactified Minkowski spacetime, $\mathbf{R}^{1} \otimes S^{3}$, approached from the $A d S_{5}$ cone. The description of the $q-(q q)^{0^{ \pm}}$system on the $\mathbf{R}^{1} \otimes S^{3}$ manifold has been executed in terms of the scalar conformal equation there, gauged by a cotangent potential. The scalar conformal equation was cast into the form of a Klein-Gordon version of the eigenvalue problem of the squared 4d angular momentum operator on $S^{3}$ and presented in eq. (10). The spectrum of such a two-body system falls as a whole into a $\infty$ d unitary representation of the conformal group, whose levels are irreps of $S O(4)$, the maximal compact group of $S O(2,4)$. For such $S O(2,4)$ irreps, the notion of "conformal bands" has been used. We observed that all nucleon resonances listed so far by the Particle Data Group distribute fairly well over the first five levels of a respective conformal band. Same applies to the $\Delta$ resonances. We identified 38 reported excitations matched by states from the predicted conformal spectrum and calculated a total of 32 resonances needed for the completeness of the two conformal $N$ and $\Delta$ bands. We did not exclude none of the resonances from the analyzes. The levels of the conformal band are constituted from parity pairs of rising spins of almost equal masses and a state of maximal spin of approximately same mass that remains as a parity simplex. In this way, more than $54 \%$ of the predicted conformal spectrum has been matched by experimentally observed states. Finding all the states belonging to the remaining $46 \%$ would provide a compelling argument in favor of the realization of conformal symmetry in QCD in the infrared. However, one should always keep in mind that the baryonic high-spin states are not stable fundamental particles but unstable composite many-body systems, which can develop a complicated internal dynamics. The latter can impose additional conditions on the observability of the "missing" states. In that regard suffices to mention threshold and cusp effects. Compared to the conformally symmetric description studied here, the quantum Hamiltonian of the real resonance systems may contain higher-order terms of one

or more different symmetries. In effect, the irreducible representations of the corresponding symmetry groups can get mixed up and suppress some of the quantum numbers. Such 
a situation in physics is by no means new. Nuclear physics provides many examples for systems in which one part of the spectrum enjoys a symmetry while the remaining part either does not, or, has an other symmetry. Recently, the concept of "Partial Dynamical Symmetry" has been developed out of the need to address this type of peculiarity of many-body excitations [56]. Within this new and more relaxed symmetry context, the degeneracy phenomenon in the observed part of the baryon spectra investigated here, already signals relevance of conformal symmetry in the light flavor baryon spectra in line with $\mathrm{AdS}_{5} / \mathrm{CFT}_{4}$.

We also illustrated quality of the wave functions in calculating realistic values for charge radii and electric-charge form factors of the proton, the $P_{11}(1440)$, and $S_{11}(1535)$ states. We furthermore observed that with the increase of the excitation energies, when the influence of the gauge potential gradually fades away, and the spectrum approaches that of the free conformal rigid rotor, the data fit becomes better. Also these observations point toward relevance of conformal symmetry for the spectra of the light flavor baryons. Conformal symmetry in the $N$ and $\Delta$ spectra is not an exact symmetry. The model presented accounts for this circumstance partly through managing the conformal constant in eq. (7) as a free parameter, and partly through the modification of the centrifugal barrier of the conformally invariant free geodesic motion on $S^{3}$ through the gauge interaction. This modification is responsible for the systematic $P_{2 I, 1}-S_{2 I, 1}$ mass splitting, which finds a satisfactory explanation within the framework under discussion. Especially the relatively large splittings of about $70 \mathrm{MeV}$ in the well established $P_{11}(1440)-S_{11}(1535)$, and $S_{11}(1650)-P_{11}(1710)$ pairs have been well reproduced. As regarding the $S_{11}(2090)-P_{11}(2100)$ pair, we reproduce correctly the mass ordering but overestimate the splitting. However, given the poor statistical knowledge on these states (one star resonances) no conclusion can be drawn from the discrepancy. The description of conformal symmetry as approximate is one of the advantages of the Klein-Gordon version (10) of the conformal equation on $S^{3}$ over its Schrödinger version in eq. (31) (earlier considered by us in ref. [52]) which keeps respecting in the interacting case the degeneracies of the free geodesic motion. Encouraging, the reasonable shape of the instantaneous effective gluon propagator obtained as a Fourier transform of the cotangent gauge potential. To recapitulate, we find conformal symmetry relevant for the spectra of the lightest baryons. 


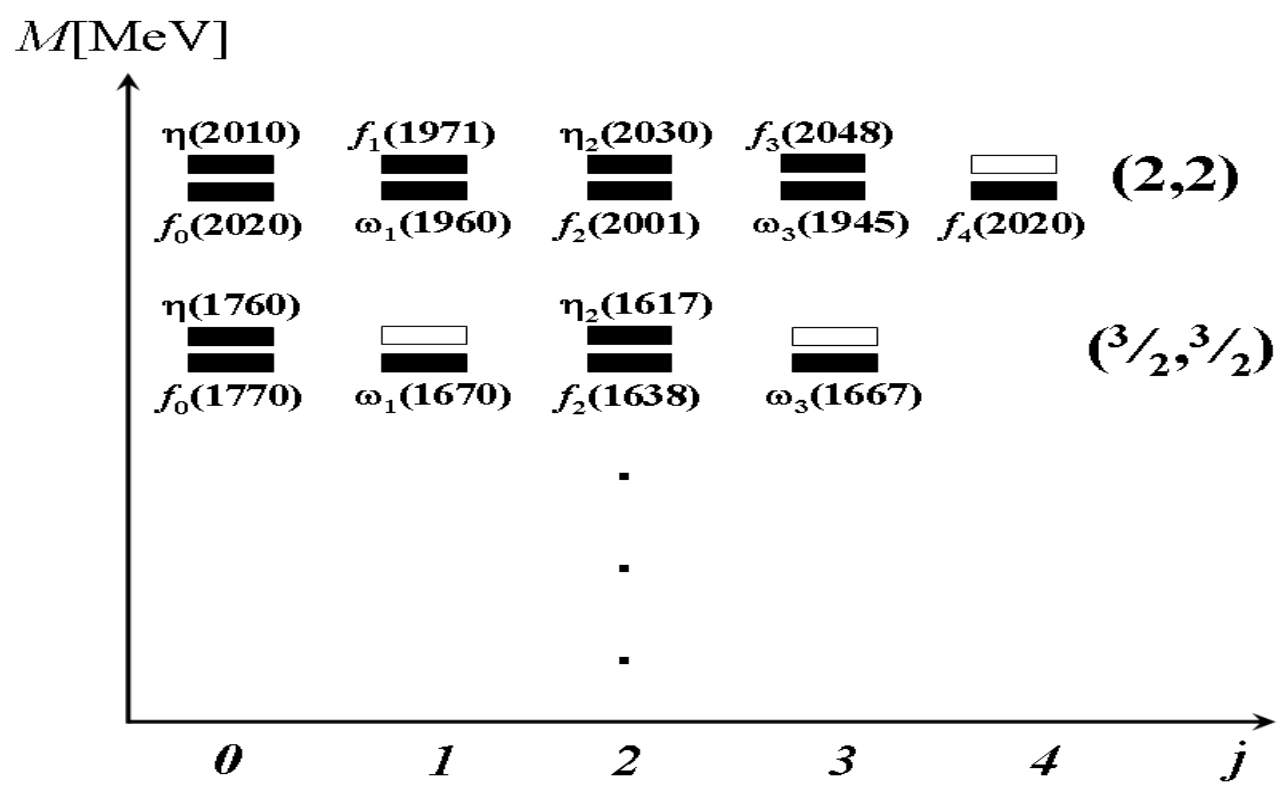

FIG. 6: Schematic spectrum of isoscalar non-strange high lying mesons according to Crystal Barrel data. Empty bricks denote "missing" states. To the right the $(K / 2, K / 2)$ levels from the conformal band with $K=3,4$ have been marked. The levels appear parity duplicated.

Finally, a comment on the relevance of the elaborated scheme for mesons is in order. In ref. [57] the Crystal Barrel data of the high-lying non-strange mesons have been analyzed and shown to be supportive of the spin-clustering phenomenon suggested in ref. [45], though the $\mathrm{SO}(4)$ levels of the conformal bands have not been explicitly constructed in ref. [57]. We here fill this gap on the example of the data below $2100 \mathrm{MeV}$ for purely illustrative purposes. The detailed analysis of the meson sector will be presented elsewhere. So far we restrict ourselves to draw the reader's attention to Figs. 6 and 7 which depict the population of the $(3 / 2,3 / 2)$ and $(2,2)$ levels of the conformal bands for isoscalar, and isovector mesons, respectively. Compared to baryons, the mesonic $S O(4)$ levels appear parity duplicated which can be read as a signal for chiral symmetry restoration from the Goldstone mode at low energies to the Wigner-Weyl mode at higher energies. Therefore, as correctly noticed in ref. [57], the Crystal Barrel data provide a clear hint on the relevance of both chiral and conformal symmetry for the lightest flavor mesons at high energies.

All in all, the model developed in the present work provides in our opinion a reasonable quantum mechanical approach to QCD which is congruent with the conformal symmetry aspect of the gravity-gauge duality. 


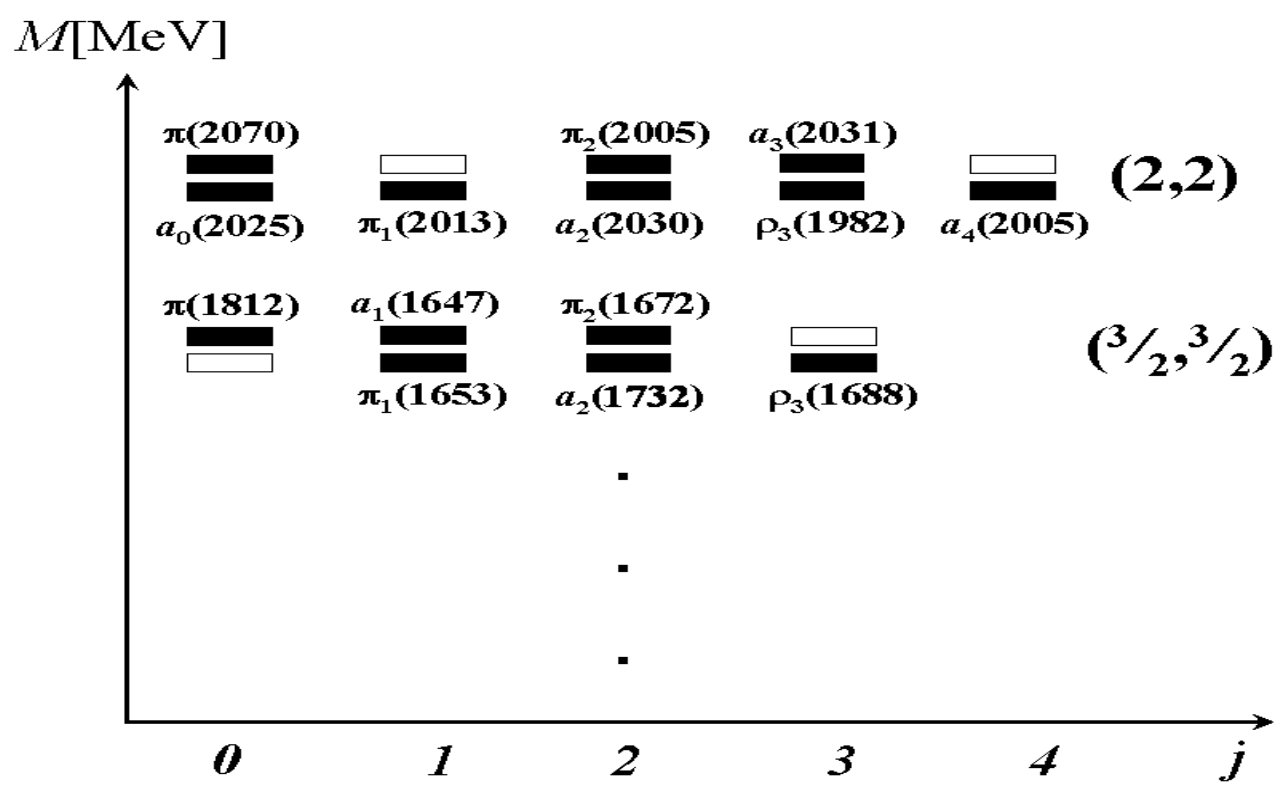

FIG. 7: Schematic spectrum of isovector non-strange high lying mesons according to Crystal Barrel data. Empty bricks denote "missing" states. To the right the $(K / 2, K / 2)$ levels from the conformal band with $K=3,4$ have been marked. The levels appear parity duplicated.

Work supported by CONACyT-México under grant number CB-2006-01/61286.

[1] V. D. Burkert, T. S. H. Lee, Electromagnetic meson production in the nucleon resonance region, Int. J. Mod. Phys. E 13, 1035-1112 (2004).

[2] S. S. Afonin, Parity doublets in particle physics, Int. J. Mod. Phys. A 22, 4537-4586 (2007).

[3] S. Capstick, N. Isgur, Baryons in a relativized quark model with chromodynamics, Phys. Rev. D 34, 2809-2835 (1986).

[4] S. G. Amsler et al., Review of particle physics, Phys. Lett. B 667, 1 (2008).

[5] R. L. Jaffe, F. Wilczek, Diquarks and exotic spectroscopy, Phys. Rev. Lett. 91, 232003 (2003); R. L. Jaffe, Exotics, Phys. Rep. 409, 1-45 (2005).

[6] E. Santopinto, Interacting quark-diquark model of baryons, Phys. Rev. C 72, 022201 (R) (2005).

[7] E. Klempt, J.-M. Richard, Baryon spectroscopy, Rev. Mod. Phys. 82, 1095 (2010).

[8] Guy F. de Téramond, Stanley J. Brodsky, The hadronic spectrum of a holographic dual QCD, 
Phys. Rev. Lett. 94, 201601 (2005);

Guy F. de Téramond and Stanley J. Brodsky, Light-front holography and gauge/gravity duality: The light meson and baryon spectra, Nucl. Phys. B, proc. Suppl. 199, 89 (2010).

[9] Stanley J. Brodsky, Guy F. de Téramond, Hadronic spectra and light-front wave functions in holographic QCD, Phys. Rev. Lett. 96, 201601 (2006);

Stanley J. Brodsky and Guy F. de Téramond, AdS/CFT and light front QCD, arXiv:0802.0514.

[10] E. Klempt, $\Delta$ resonances: quark models, chiral symmetry and AdS/QCD, Eur. Phys. J. A 38, 187-194 (2008).

[11] Jaemo Park, Piljin Yi, A holographic QCD and excited baryons from string theory, J. High Enery Phys. 06 (2008) 011.

[12] K. Nawa, H. Suganama, T. Kojo, Brane-induced Skyrmion on $S^{3}$ : Baryonic matter in holographic QCD, Phys. Rev. D 79, 26005 (2009).

[13] Koji Hashimoto, Holographic nuclei, J. High Energy Phys. 12 (2009) 065.

[14] H. Nicolai, E. Sezgin, Y. Tanii, Conformally invariant supersymmetric field theories on $S^{p} \times S^{1}$ and super p-branes, Nucl. Phys. B 305, 483-496 (1988).

[15] M. Lüscher, G. Mack, Global conformal invariance in quantum field theory, Commun. Math. Phys. 41, 203-234 (1975).

[16] Gary T. Horowitz, Hirosi Ooguri, Spectrum of large N gauge theory from supergravity, Phys. Rev. Lett. 80, 4116-4118 (1998).

[17] G. W. Gibbons, A. R. Steit, Sphalerons and conformally compactified Minkowski space, Phys. Lett. B 346, 255-261 (1995).

[18] S. Ferrara, Ch. Fronsdal, Conformal Maxwell theory as singleton field theory on $A d S_{5}$, IIB three branes and duality, Class. Quant. Grav. 15, 2153-2164 (1998).

[19] J. Maldacena, Wilson loops in large N field theories, Phys. Rev. Lett. 80, 4859-4862 (1998).

[20] Nathan Seiberg, Field Theory:The past 25 Years, http://www.sns.ias.edu/ seiberg/talks.htm

[21] C. W. Gibbons, P. K. Townsend, Vacuum interpolation in supergravity via super p-brane, Phys. Rev. Lett. 71, 3754-3757 (1993).

[22] Jan de Boer, Liat Maoz, Asad Naqvi, Some aspects of AdS/CFT correspondence arXiv:hep-th/0407212.

[23] Harald Dorn, Christoph Sieg, Conformal boundary and geodesics for $A d S_{5} \times S^{5}$ and the plane 
wave: Their approach in the Penrose limit, JHEP 0304:030 (2003).

[24] Vijay Balasubramanian, Per Kraus, A stress tensor for Anti-DeSitter gravity, Commun. Math. Phys. 208, 413-428 (1999).

[25] J. L. F. Barbon, C. A. Fuertes, On the spectrum of non-relativistic AdS/CFT, JHEP 0809:030 (2008).

[26] M. P. Dabrowski, J. Garecki, D. B. Blaschke, Conformal transformations and conformal invariance in gravity, Ann. d. Physik 18, 13-22 (2009).

[27] Y. S. Kim, M. E. Noz, Theory and application of the Poincaré group (D. Reidel, Dordrecht, 1986).

[28] Brian G. Wybourne, Classical groups for physicists (Wiley-Inter-science, N.Y., 1974).

[29] R. R. Aldinger, A. Bohm, P. Kielanowski, M. Loewe, P. Magnollay, N. Mukunda, Relativistic rotator.I. Quantum observables and constrained Hamiltonian mechanics, Phys. Rev. D 28 3020-3031 (1983).

[30] A. V. Anisovich et al, Quark-diquark systematics of baryons and the SU(6) symmetry of light states, arXiv:1002.1777[hep-ph].

[31] Sheldon Axler, Paul Bourdon, Wade Ramey, Harmonic Function Theory, (Springer Verlag N.Y.Inc, 2001)

[32] E. Schrödinger, A method of determining quantum mechanical eigenvalues and eigenfunction, Proc. Roy. Irish Acad. A 46, 9-16 (1940).

[33] P. W. Higgs, Dynamical symmetries in a spherical geometry, J. Phys A:Math.Gen. 12, 309-323 (1979).

[34] C. B. Compean, M. Kirchbach, The trigonometric Rosen-Morse potential in the supersymmetric quantum mechanics and its exact solutions, J. Phys. A:Math.Gen. 39, 547-557 (2006).

[35] C. B. Compean, M. Kirchbach, Trigonometric quark confinement potential of QCD traits, Eur. Phys. J. A 33, 1-4 (2007).

[36] A. O. Barut, Raj Wilson, On the dynamical group of the Kepler problem in a curved space of constant curvature, Phys. Lett. A 110, 351-354 (1985).

[37] Gary T. Horowitz, A. A. Tseytlin, On exact solutions and singularities in string theories, Phys. Rev. D 50, 5204 (1994).

[38] G. Papadopoulos, J. G. Russo, A. A. Tseytlin, Curved branes from string dualities, Class. Quantum Grav. 17, 1713-1728 (2000). 
[39] E. Eichten, H. Gottfried, T. Kinoshita, K. D. Lane, T.M. Yan, Charmonium:The model, Phys. Rev. D 17, 3090-3117 (1978);

E. Eichten, H. Gottfried, T. Kinoshita, K. D. Lane, T.M. Yan, Charmonium:Comparison with experiment, Phys. Rev. D 21, 203-233 (1980).

[40] T. T. Takahashi, H. Suganuma, Y. Nemoto, H. Matsufuru, Detailed analysis of the three-quark potential in SU(3) lattice QCD, Phys. Rev. D 65, 114509-1-19 (2002).

[41] Wen-Yu Wen, Multi-quark potential from ADS/QCD, Int. J. Mod. Phys. 123, 4533-4543 (2008);

H. Boshi-Filho, N. R. F. Braga, C. N. Ferreira, Static strings in Randall-Sundrum scenarios and the quark-antiquark potential, Phys. Rev. D 73, 106006 (2006) ;

Ghanghyun Ahn, J. F. Vázquez-Portiz, From marginal deformation to confinement, J. High Energy Phys. 06(2006) 061.

[42] N. Brambilla, A. Vairo, Th. Rosch, Effective field theory Lagrangians for baryons with two and three heavy quarks, Phys.Rev.D 72, 034021 (2005);

N. Brambilla, Effective Field Theories for $Q Q Q$ and $Q Q q$ baryons, AIP Conf.Proc.756,366$368(2005)$.

[43] A. Raposo, H.-J. Weber, D. E. Alvarez-Castillo, M. Kirchbach, Romanovski polynomials in selected physics problems, C. Eur. J. Phys. 5, 253-284 (2007).

[44] C. B. Compean, M. Kirchbach, Momentum space trigonometric Rosen Morse potential, J. Phys. A:Math.Theor. 42, 365301 (2009).

[45] M. Kirchbach, On the parity degeneracy of baryons, Mod. Phys. Lett. A 12, 2373-2386 (1997).

[46] M. Kirchbach, Classifying reported and "missing" resonances according to their $P$ and $C$ properties, Int. J. Mod. Phys. A 15, 1435-1451 (2000).

[47] M. Kirchbach, M. Moshinsky, Yu. F. Smirnov, Baryons in O(4) and vibron model, Phys. Rev. D 64,114005 (2001).

[48] M. Kirchbach, Degeneracy symmetry of baryon spectra, Nucl. Phys. A 689 157-1666 (2001).

[49] T. Melde, K. Berger, L. Canton, W. Plessas, R. F. Wagenbrunn, Electromagnetic nucleon form factors in instant and point form, Phys. Rev. D 76, 074020 (2007).

[50] K. Nagata, A. Hosaka, Sructure of the nucleon and the Roper resonance with diquark correlations, Mod. Phys. Lett. A 23, 2397-2400 (2008).

[51] Jarl Nissfolk, Tobias Ekholm, Christer Elvingson, Brownian dynamics simulations on a hy- 
persphere in 4 space, J. Chem. Phys. 119, 6423-6432 (2003).

[52] M. Kirchbach, C. B. Compean, Baryons from quarks in color gauge space of constant positive curvature and deconfinement, arXiv:0805.2404][hep-ph].

[53] P. O. Fedosenko, Infrared behavior of gluon and ghost propagators in $Q C D$ in the Landau gauge, Ukr. J. Phys. 52, 607-617 (2007).

[54] A. C. Aguilar, D. Binosi, J. Papavassiliou, Gluon and ghost propagators in the Landau gauge: Deriving lattice results from Schwinger-Dyson equations, Phys. Rev. D 78, 025010 (2008).

[55] C. Alexandrou, Ph. de Forcand, E. Follana, Gluon propagator without Gribov copies on a finer lattice, Phys. Rev. D 65, 114508 (2002).

[56] J. E. Garcia-Ramos, A. Leviatan, P. Van Isacker, Partial Dynamical Symmetry in Quantum Hamiltonians with Higher-Order Terms, Phys. Rev. Lett. 102, 112502 (2009).

[57] S. S. Afonin, Light meson spectrum and classical symmetries of QCD, Eur. Phys. J. A 29, 327-335 (2006). 